\title{
Potential Biomarkers for Predicting the Overall Survival of Kidney Renal Papillary Cell Carcinoma: A Analysis of N6-Methylandenosine-Related IncRNAs
}

\section{zixuan Wu}

Guangzhou University of Traditional Chinese Medicine: Guangzhou University of Chinese Medicine

\section{Xuyan Huang}

Guangzhou University of Traditional Chinese Medicine: Guangzhou University of Chinese Medicine Min-jie Cai

Guangzhou University of Traditional Chinese Medicine: Guangzhou University of Chinese Medicine

Peidong Huang ( $\sim$ yeruyun@163.com )

Yunnan University of Traditional Chinese Medicine https://orcid.org/0000-0002-3044-9269

\section{Research}

Keywords: Breast conservative surgery, mastectomy, breast cancer, cancer surgery, India

Posted Date: November 15th, 2021

DOl: https://doi.org/10.21203/rs.3.rs-1052018/v1

License: (c) (i) This work is licensed under a Creative Commons Attribution 4.0 International License. Read Full License 


\section{Abstract}

Background: It is well established that disease-free survival and overall survival after breast conservation surgery (BCS) followed by radiotherapy are equivalent to that after mastectomy. However, in Asian countries, the rate of BCS continues to remain low. The cause may be multifactorial including the patient's choice, availability and accessibility of infrastructure and surgeon's choice. We aimed to elucidate the Indian surgeons' perspective while choosing between BCS and mastectomy, in women oncologically eligible for BCS.

Methods: We conducted a survey-based cross-sectional study over 3 weeks between January-February 2021. Indian surgeons with general surgical or specialised onco-surgical training, who consented to participate were included in the study. Multinomial logistic regression was performed to assess the effect of study variables on offering mastectomy or BCS to an eligible patient.

Results: A total of 347 responses were included. The mean age of the participants was $43(11)$ years. $63 \%$ of the surgeons were in the $25-44$ years age group with the majority ( $80 \%$ ) being males. $66.4 \%$ of surgeons 'almost always' offered BCS to oncologically eligible patients. Surgeons who had undergone specialised training in oncosurgery or breast conservation surgery were 35 times more likely to offer BCS $(p<0.01)$. Surgeons working in hospitals with in-house radiation oncology facilities were 9 times more likely to offer BCS $(p<0.05)$. Surgeons' years of practice, age, sex and hospital setting did not influence the surgery offered.

Conclusion: Our study found that two-thirds of Indian surgeons preferred BCS over mastectomy. Lack of radiotherapy facilities and specialised surgical training were deterrents to offering BCS to eligible women.

\section{Introduction}

Kidney cancer is becoming a more widespread cancer kind all around the world ${ }^{\mathrm{D}}$. It is a different most frequent genitourinary system tumor in China, with an annual increase ${ }^{\square}$. Kidney Renal papillary cell carcinoma (KIRP), which accounts for $15-20 \%$ of all kidney cancers, develops from the proximal nephron, the same location as clear cell carcinoma ${ }^{\square}$. KIRP, on the other hand, is regarded as a more heterogeneous disease in terms of disease development and patient survival outcomes ${ }^{\square}$. KIRP can be treated in a variety of ways, including surgery, radiation, and chemotherapy, however these only provide a minimum benefit ${ }^{[-]}$. Due to the small number of instances. KIRP patients are typically excluded from genetic studies and randomized therapeutic trials for kidney cancer ${ }^{\square}$. Nonetheless, a lack of accurate biomarkers for early tumor diagnosis, as well as limited preclinical models, has hampered effective KIRP therapeutic therapy ${ }^{[-]}$. Additional molecular identification is critical for both fundamental and clinical 
investigations of KIRP, as well as the identification of novel and effective KIRP prognostic biomarkers, in order to prevent the initial start and progression of KIRP.

$\mathrm{M}^{6} \mathrm{~A}$ is not only involved in RNA splicing, output, and translation, but it is also the most common internal epistemic modification of mRNA and IncRNAs ${ }^{[-]}$. $\mathrm{M}^{6} \mathrm{~A}-\mathrm{IncRNAs}$ play an important function in the regulation of several biological and pathological processes ${ }^{\square}$. The $\mathrm{m}^{6} \mathrm{~A}$-regulators, which include methyltransferase complexes ("writers"), signal transducers ("readers"), and demethylase complexes ("erasers") $)^{\square}$. In a number of studies, the amount of the $\mathrm{m}^{6} \mathrm{~A}$ regulatory factor has been associated with chemotherapy sensitivity, the rate of tumor stem cell self-renewal, and the rate of cancer cell proliferation ${ }^{[-]}$. Because abnormal $\mathrm{m}^{6} \mathrm{~A}$ mutations may promote tumor growth by increasing tumor stem cell self-renewal ${ }^{\square}$, researchers created a novel treatment strategy: Restoring RNA methylation balance in tumor cells ${ }^{[-]}$. Many IncRNAs are thought to be risk factors for the prognosis of KIRP patients, and they have a role in the incidence of various cancers. KIRP is related to the occurrence and progression of $\mathrm{m}^{6} \mathrm{~A}$ IncRNAs, which are partially overexpressed in tumor tissues ${ }^{[-]}$. Immune checkpoint-related gene profiles may be helpful in assessing risk, predicting survival, and identifying treatment responsiveness in KIRP patients ${ }^{\square}$. Despite the fact that little research has been conducted on the relationship between $\mathrm{m}^{6} \mathrm{~A}$ related IncRNAs and immune cell infiltration in KIRP, it is critical to investigate immune cell infiltration in the tumor microenvironment (TME) and its relationship with KIRP clinicopathological aspects of tumor. At this time, the cause and mechanism of KIRP's abnormal IncRNA expression and $\mathrm{m}^{6} \mathrm{~A}$ methylation are unknown. It is critical to create transcriptional maps of IncRNA expression and $\mathrm{m}^{6} \mathrm{~A}$ alteration in KIRP patients in order to identify the IncRNA-related pathways that affect KIRP patients' prognosis. Immune checkpoint-related gene profiles can be used to assess risk and predict overall survival in KIRP patients by predicting therapeutic responsiveness. Understanding how $\mathrm{m}^{6} \mathrm{~A}$-IncRNAs modification contributes to KIRP progression may lead to the identification of a biomarker that may be invoked as a therapeutic target.

The objective of this study was to identify $\mathrm{m}^{6} \mathrm{~A}$-related IncRNAs whose expression is linked to the prognosis of KIRP patients in a predictive model designed to make KIRP prognosis prediction easier. To better understand $\mathrm{m}^{6} \mathrm{~A}$-Incrna infiltration and their associated immune cells in TME, and to aid in the discovery of new therapeutic targets and pharmaceutical possibilities for KIRP therapy.

\section{Marerials And Methods}

\subsection{Datasets and $\mathrm{m}^{6} \mathrm{~A}$-Related Genes}

Using the Genomic Data Commons Data Portal (https://portal.gdc.cancer.gov/) D, we gathered KIRP gene expression patterns and clinical data from the Cancer Genome Atlas (TCGA). The method is based on gene expression profiles (Cases: Kidney and TCGA and TCGA-KIRP; Files: transcriptome profiling and Gene Expression Quantification and HTSeq-FPKM). Clinical research information (Cases: Kidney and TCGA and TCGA-KIRP; Files: Clinical and bcr xml). The expression patterns of 298 cases of KIRP and 32 
cases of normal tissues were included in the TCGA public database on October 6, 2021. Furthermore, expression data for writers (METTL3, METTL14, METTL16, WTAP, VIRMA, ZC3H13, RBM15 and RBM15B), readers (YTHDC1, YTHDC2, YTHDF1, YTHDF3, HNRNPC, FMR1, LRPPRC, HNRNPA2B1, IGFBP1, IGFBP2, IGFBP3, and RBMX) and erasers (FTO and ALKBH5)

\subsection{Annotation of IncRNAs}

The Genome Reference Consortium Human Build 38 (GRCh38) long non-coding RNA annotation file was retrieved from the GENCODE website ${ }^{4}$ for annotation of the IncRNAs in the TCGA dataset. Using Perl software (https://www.perl.org/), the transcriptome data and human configuration files were matched and sorted, and the proper mRNA and IncRNA gene expression data were obtained. The gene IDs were translated to gene names using information from the ensemble database (http://asia.ensembl.org/info/data/index.html). The Limma package of R4.1.0 was used to extract $\mathrm{m}^{6} \mathrm{~A}$ related gene expression data based on the previously gathered gene expression matrix of $\mathrm{m}^{6} \mathrm{~A}$-related IncRNA gene expression profile information.

\subsection{Identification of $\mathrm{m}^{6} \mathrm{~A}$-related IncRNAs}

The Limma package's correlation test was used to evaluate the expression of $\mathrm{m}^{6} \mathrm{~A}$-related IncRNA after excluding the normal samples and using $p<0.001$ and corFilter $=0.4$ as screening criteria. The link between $\mathrm{m}^{6} \mathrm{~A}$-related gene expression and IncRNAs was discovered using co-expression analysis. In order to demonstrate the link intuitively, the network of IncRNAs associated with $\mathrm{m}^{6} \mathrm{~A}$ is visualized using the igraph software. Limma was used to connect $\mathrm{m}^{6} \mathrm{~A}$-related IncRNA expression data with clinical survival data, and survival was utilized to perform university cox analysis. Following visualization, the confidence interval and the risk ratio was determined, and the forest diagram was constructed. Limma, pheatmap, reshape2, and ggpubr programs were used to compare differences in $\mathrm{m}^{6} \mathrm{~A}$-related prognostic genes between normal and malignant tissues. In order to directly reflect the difference table of $\mathrm{m}^{6} \mathrm{~A}$-related

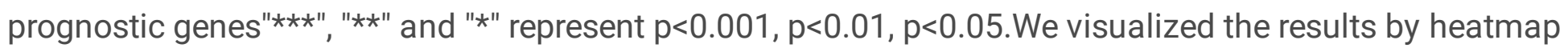
and boxplot.

\subsection{Role of $\mathrm{m}^{6} \mathrm{~A}$-related IncRNAs}

First, we performed cluster analysis using Limma and ConsensusClusterPlus programs, and we divided the prognosis-related $\mathrm{m}^{6} \mathrm{a}$-Incrna into two subtypes: cluster 1 and cluster 2 . The Survminer package was used to investigate the survival of IncRNA subtypes, and the survival package was used to evaluate the prognostic value of $\mathrm{m}^{6} \mathrm{a}-$ Incrna. The differential expression of prognosis-related IncRNAs in each cluster was shown as a heatmap using the pheatmap tool, and the relationship between IncRNAs and clinicopathological features was investigated. The limma program was used to identify differences in the expression of target genes of the relevant subtype and distinct types of tissue. Refer to the Uniprot database $^{\square}$ (https://www.uniprot.org/) to standardize the standard name of the target gene. The gene correlation of target gene and prognostic $\mathrm{m}^{6} \mathrm{~A}$-related IncRNAs in KIRP was analyzed by Limma and 
corrplot packages. When the $p<0.05$, the difference was statistically significant, and the results was visualized by corrplot.

\subsection{Role of immune cell infiltration and the TME and the predictive nomogram}

We investigated and computed the infiltration of various immune cells in tissue samples using preprocessCore, limma, and the e1071 package, and discovered that there was some immune cell infiltration. After analyzing the TME with the ESTIMATE and limma packages, the stromalscore, immunescore, and ESTIMATE score were generated. Tumor purity was found to be inversely related to the above-mentioned scores. The limma package was used to investigate differences in immune cell infiltration among different clusters, and vioplot was used to show the results. Simultaneously, the limma package was used to assess the infiltration of each type of immune cell in different KIRP clusters, and the results are shown as a boxplot. Following that, we utilized the limma package to examine the differences in TME in different subtypes of the samples based on the immunological score, ESTIMATE score, and stroma score, and matching boxplots were constructed to assess the purity of tumor cells in the various TME subtypes. GSEA (https://www.gsea-msigdb.org/gsea/index.jsp) was utilized to find differences in linked functions and pathways in several samples, and data was imported using the PERL programming language. Associated score and graphs were used to determine the functions and routes in different clusters were dynamic (c2.cp.kegg.v.7.2.symbols.gmt,cluster.cls\#C2 versus C1). Each sample was identified as 'H' or 'L' depending on whether it was a high-risk cluster of prognosis-related IncRNAs. The number of permutations, no collapse, and phenotype were all set to 1000 , no collapse, and phenotypic. The gene list was sorted in 'real' mode, with the genes listed in descending order. The 'Signal2Noise' measure was used to rank the genes. The normalization method was 'meandiv,' and the difference was statistically significant with a FDR $<0.05$. A nomogram was constructed integrating the prognostic signatures, for predictive of 1,2 and 3 year OS of KIRP patients.

\subsection{Development of the $\mathrm{m}^{6} \mathrm{~A}-\mathrm{InCRNA}$-related prognostic model}

Lasso regression was utilized to create a prognostic model. All of the samples were segregated into two groups based on the median value of the risk score of the prognostic $\mathrm{m}^{6} \mathrm{~A}-\mathrm{IncRNAs}$ : high-risk and lowrisk. The training (50\%) and test (50\%) groups were identified in Lasso regression. And the appropriate plots were obtained. The survival curves for the high-risk and low-risk groups were constructed and compared. To examine the accuracy of our model in predicting survival in KIRP, a comparable receiveroperating characteristics (ROC) curve was set up using the timeROC software. A risk curve depended on on the risk score, and the survival status and risk associated with $\mathrm{m}^{6} \mathrm{~A}-\mathrm{IncRNAs}$ were analyzed in respect to the curve. An independent prognostic analysis was conducted to see whether our model was independent of other clinical prognostic factors that could influence the patients' outcome. The researchers generated hazard ratios using multivariate and university models. We employed model validation for clinical groups to assess and validate whether our model could be applicable to a variety of 
clinical groups. To distinguish between high-risk and low-risk $\mathrm{m}^{6} \mathrm{~A}-\mathrm{IncRNAs}$ and to investigate the relationship between clinical variables and our prediction risk model. Analyses of risk and clinical connection were out. The heatmap was created using the pheatmap and limma programs. Boxplots of risk and clinical relevance were obtained to estimate risk based on clinical data. The changes in target gene expression in our model's different risk groups for KIRP patients were evaluated using genetic difference analysis. A correlation study of the risk and immune cells was conducted and shown as a scatterplot to assess the relationship between the immune cells and the risk score. To further demonstrate the correctness of our model, Decision Curve Analysis (DCA) was constructed.

\section{Results}

The purpose of this study was to demonstrate how $\mathrm{m}^{6} \mathrm{~A}-\mathrm{IncRNAs}$ and immune cell infiltration alter KIRP. We detected $32 \mathrm{~m}^{6} \mathrm{~A}$-related prognostic IncRNAs and 3 high-risk $\mathrm{m}^{6} \mathrm{~A}$-IncRNAs based on differences in expression between tumor and normal tissues. GSEA was utilized to identify latent signaling pathways that could be implicated in the development and progression of KIRP, and lasso regression was used to generate a suitable predictive model. The TME was utilized to evaluate and calculate the infiltration of different immune cells in the samples.

\subsection{Identification of $\mathrm{m}^{6} \mathrm{~A}$-related IncRNAs}

To discriminate between mRNA and IncRNA, the $\mathrm{m}^{6} \mathrm{~A}$-related gene expression data were retrieved from the aggregated transcriptome data. To determine the relationship between $\mathrm{m}^{6} \mathrm{~A}$-related gene expression and IncRNAs, a network plot was created (Figure.1a). A forest plot depicts the results of univariate Cox regression analysis (Figure. $1 b$ ). When $p<0.05$, the IncRNAs were considered to be $m^{6} A$-related prognostic IncRNAs. Variations in expression of $\mathrm{m}^{6} \mathrm{~A}$-related prognostic IncRNAs between tumor and normal tissues were discovered and displayed as a heatmap and boxplot (Figures.1c-d). The expression of $32 \mathrm{~m}^{6} \mathrm{~A}-$ related prognostic IncRNAs changed between tumors and normal tissues. Some IncRNAs were considered to be highly expressed in tumors, while others were found to be strongly expressed in normal tissues $(p<0.05)$.

\subsection{Role of $\mathrm{m}^{6} \mathrm{~A}-\mathrm{InCRNAs}$}

When $\mathrm{K}=2$, there was the least overlap between the two types, and the CDF value was the lowest; thus, we divided IncRNAs into two types: cluster 1 and cluster 2. A survival analysis was done by utilizing the IncRNA subtypes to determine the prognostic value of $\mathrm{m}^{6} \mathrm{~A}-\mathrm{IncRNAs}$, and the survival rate of cluster 1 was higher than that of cluster $2(\mathrm{P}<0.001)$, as showed in Figure.2a. A heatmap was used to represent differences in the expression of prognosis-related IncRNAs, and the relationship was studied in relation to clinicopathological features (Figure.2b). Some IncRNAs were found to be highly expressed in cluster 1 . Whereas others were shown to be strongly expressed in cluster 2. Although prognosis-associated IncRNAs were related to age, gender, and grade $(P<0.05)$, there was no difference in their expression across clusters, as showed in Figure.2b. Differences in target gene expression in related subtypes and 
other types of tissue specimens are shown in Figures.2c-d. FANCL expression was lower in normal tissue than in malignant tissue $(\mathrm{P}<0.001)$, although the above-mentioned gene was more expressed in cluster 2 $(p<0.01)$, which means that FANCL might be an oncogene for KIRP. We conducted gene correlation analysis to investigate if there was any relationship between the target gene and prognosis $\mathrm{m}^{6} \mathrm{~A}-\mathrm{IncRNAs}$ in KIRP (Figure.2e), and we discovered that the target gene is associated with prognostic $\mathrm{m}^{6} \mathrm{~A}-\mathrm{In} \mathrm{n}$ RNAs $(P<0.05)$.

\subsection{Role of immune cell infiltration and TME}

A vioplot was created by analyzing the differences in immune cell infiltration in distinct clusters (Figure.3a). The infiltration of each type of immune cell in various KIRP clusters was studied and represented as a boxplot. (Figure.3b-i); Immune cells, such as Plasma cells, Macrophages M0, Macrophages M2 were highly clustered in cluster $1(p<0.05)$, whereas B cells naive, T cells CD8, T cells CD4 naive, T cells CD4 memory activated, T cells follicular helper, T cells regulatory (Tregs), NK cells resting, Macrophages $M 1$, and Dendritic cells activated were highly clustered in cluster $2(p<0.05)$; there was no intergroup difference for the other cells that were analyzed ( $p>0.05)$. We compared the TME in different sample subtypes, and matching boxplots were created to further investigate the purity of tumor cells in various specimen types (Figure.3m-o). Cluster 1 has higher scores across the board, indicating lower tumor cell purity and more immune-related cells in the TME $(p<0.05)$. Then, to elucidate the differences in linked function and pathways in different samples, GSEA was used (Figure.4). Each cluster's top 6 enriched functions or pathways are presented. The FDR q-value and FWER p-value were all $<0.05$. The 'P53 SIGNALING PATHWAY', as a result, was the most enriched pathway, and some of them were positively associated with $\mathrm{C} 1$ or $\mathrm{C} 2$.

\section{$3.4 \mathrm{~m}^{6} \mathrm{~A}$-IncRNA-related prognostic model}

The median value of the risk score of prognostic $\mathrm{m}^{6} \mathrm{~A}-$-IncRNAs was utilized to split all samples into highrisk and low-risk groups using lasso regression. In lasso regression, there were training (50\%) and test (50\%) groups, and associated plots were created (Figure.5a-b). The survival curves for the high-risk and low-risk groups were compared (Figure.5c-d). The survival rate of the low-risk subtype was greater than of the high-risk subtype in both the test and training groups $(p<0.05)$. A corresponding ROC curve was obtained using the timeROC package to evaluate the accuracy of our model in predicting the survival of patients with the disease (Figure.5e-f), where both the areas under the curve (AUCs) were $>0.5$, attesting to the considerable accuracy of our model in predicting survival with the disease. Meanwhile, the signature IncRNAs' AUC was 0.828, indicating that they outperformed standard clinicopathological characteristics in predicting KIRP prognosis (Figure.6a-b). We discovered that the patient's risk score was inversely proportional to the survival of KIRP patients using a patient's risk survival status plot. Surprisingly, the majority of the novel IncRNAs identified in this research exhibited a negative relationship with our risk model, indicating that more research is needed (Figure.6c-d). For 1, 2, and 3 year survival rates, the AUC predictive value of the unique IncRNAs signature was $0.828,0.879$, and 0.837 , respectively (Figure.6e). Risk-related heatmap: ${ }^{6}$ A-IncRNAs that such as LUCAT1, FOXD2-AS1, and AC099850.4 were highly expressed in test and train group, which means all of them might be detrimental to the prognosis of KIRP 
patients (Figure.6f-g). As the risk score rises, so takes care of the number of deaths and the proportion of those at high risk. The $\mathrm{m}^{6} \mathrm{~A}$ prognosis-related IncRNAs had a significant risk. An independent prognostic study was carried out to evaluate whether our model was independent of other clinical prognostic factors that might influence the patients' outcome. COX analysis in the test group revealed that IncRNA signature (HR: $2.973,95 \mathrm{Cl}: 1.706-5.183)$, Stage (HR: $1.930,95 \mathrm{Cl}$ : 1.212-3.074). In train group, IncRNA signature (HR: 1.440, 95Cl: 1.166-1.777), Stage (HR: 2.302, 95Cl: 1.412-3.753), and Gender (HR: 2.918, 95Cl: 1.1327.522). Stage and risk score are all independent predictive risk variables for KIRP $(p<0.05)$ (Figure.7). We performed model validation for clinical groups to test and validate whether our model could be applied to different clinical groups (Figure.8), and we discovered that it could be applied to groups stratified by sex, stage, T stage, $M$ stage, and $N$ stage $(p<0.05)$. To identify high-risk and low-risk $m^{6} A-I n c R N A s$ and to investigate the relationship between clinical variables and our prediction risk model (Figure.9a-f). There was risk and clinical correlation assessments performed. We employed genetic difference analysis to examine differences in the expression of target genes in different risk groups of our model in KIRP (Figure.9g). FANCL expression was higher in the high-risk group ( $p=0.0009)$. The hybrid nomogram (Figure.10) integrating clinicopathological features and the novel $\mathrm{m}^{6} \mathrm{~A}-\mathrm{IncRNAs}$ prognostic signature was stable and accurate, and hence may be employed in KIRP patient care.

\subsection{Immunity and gene expression}

The scatterplot depicts this association and aid in assessing whether immune cells are beneficial or harmful (Figure.11). Dendritic cells resting, Macrophages M1, NK cells resting, T cells CD4 memory activated, $T$ cells follicular helper, $T$ cells regulatory (Tregs) were found to be positive linked with risk score $(R=0.2$ and $p=0.0073 ; R=0.3$ and $p=4.8 e-05 ; R=0.19$ and $p=0.012 ; R=0.25$ and $p=0.001 ; R=0.18$ and $p=0.019 ; R=0.2$ and $p=0.007)$. Macrophages $M 2$ was found to be negative linked with risk score $(R=-0.41$, $\mathrm{p}=2.7 \mathrm{e}-08$ ). Given checkpoint inhibitor-based immunotherapies are just as important, we investigated into the differences in immune checkpoint expression between the two groups. Between the two groups of patients, we discovered a significant discrepancy in the expression of TNFRSF4, TNFRSF9, TNFSF14, TNFRSF18, CD70 and other genes (Figure.12).

\section{Discussion}

Because of its advanced stage and dismal prognosis, treating KIRP is a serious clinical challenge $\mathrm{I}^{\square} \cdot \mathrm{M}^{6} \mathrm{~A}$ mutations have an effect on IncRNA processing. It regulates cellular proliferation and maturation, both of which are associated with cancer formation ${ }^{\square}$. The activation and inhibition of tumor-related signaling pathways in the urinary tract were thought to be tightly linked to $\mathrm{m}^{6} \mathrm{~A}$ regulators ${ }^{\square}$. As a result, inhibiting the $\mathrm{m}^{6} \mathrm{~A}$ mutation in cancer cells could have a broad impact on cancer research ${ }^{\square}$. In recent years, there has been many interest in the interaction hypothesis between $\mathrm{m}^{6} \mathrm{~A}$ and IncRNAs${ }^{\square}$. These findings demonstrate that the $\mathrm{m}^{6} \mathrm{~A}-\mathrm{IncRNA}$ regulation network plays a role in tumor cell proliferation, invasion, and metastasis, pointing to innovative cancer diagnostic and therapeutic targets. Therefore, we believe that further investigation of the molecular process of IncRNA and $\mathrm{m}^{6} \mathrm{~A}$ mutual regulation, as well as a better 
understanding of the relationship between the $\mathrm{m}^{6} \mathrm{~A}$ regulatory molecule and target gene RNA modification, will be a hotspot for the discovery of prognostic markers and therapeutic targets in malignant tumors.

In this study, we retrieved $\mathrm{m}^{6} \mathrm{~A}$-related gene expression data and differentiated between mRNA and IncRNA. The link between $\mathrm{m}^{6} \mathrm{~A}$-related gene expression and IncRNAs was investigated using coexpression analysis. We discovered a phenomenon in the co-expression network plot in which numerous IncRNAs were associated with $\mathrm{m}^{6} \mathrm{~A}$-related genes in KIRP. The confidence interval and hazard ratio were determined using data on prognosis-related IncRNAs. M ${ }^{6} \mathrm{~A}$-related IncRNAs were discovered to be closely connected with the prognosis of the KIRP in a university Cox regression study. This study discovered 32 $\mathrm{m}^{6} \mathrm{~A}-\mathrm{IncRNAs}$ that are associated with prognosis and expresses differently in tumor and normal tissues. Some IncRNAs have been discovered to be overexpressed in tumors. Whereas others have been found to be overexpressed in normal tissue. GPRIN1 was shown to be overexpressed in KIRP, and it was linked to a poor prognosis in a pan-cancer study. GPRIN1 was shown to be overexpressed in KIRP, and it was linked to a poor prognosis in a pan-cancer study ${ }^{\square}$. Ruyi He ${ }^{\square}$ discovered that GAS6-AS1 expression was also upregulated in kidney cancer; nevertheless, KIRP patients in the high-expression group of GAS6-AS1 had better overall survival than those in the low-expression group. The $\mathrm{m}^{6} \mathrm{~A}$ modification of IncRNAs serves a vital role in altering IncRNA structure and regulating their interaction with proteins by monitoring gene transcription inhibition and modifying its subcellular distribution ${ }^{\square}$. Despite the fact that there has been little investigation into $\mathrm{m}^{6} \mathrm{~A}-\mathrm{InRNA}$ and the KIRP. Built on the findings presented above, we may conclude that a change in the $\mathrm{m}^{6} \mathrm{~A}-\mathrm{IncRNAs}$ is linked to the onset and progression of KIRP. The results of our investigation, which reveal that multiple $\mathrm{m}^{6} \mathrm{~A}$-IncRNAs are overexpressed in tumors but not in normal tissue, are well accounted for by the previous studies. $\mathrm{M}^{6} \mathrm{~A}-\mathrm{In}$ CRNAs can function as tumor suppressors or oncogenes.

We investigated the involvement of $\mathrm{m}^{6} \mathrm{~A}-\mathrm{IncRNAs}$ in KIRP in greater depth. The predictive importance of $\mathrm{m}^{6} \mathrm{~A}$-IncRNAs was determined using survival analysis based on IncRNA subtypes. Low-risk IncRNAs improve the prognosis of KIRP patients. Furthermore, IncRNAs have been linked to patient outcomes in KIRP. Because most of the $\mathrm{m}^{6} \mathrm{~A}$-IncRNAs in our study were minimally expressed in KIRP, there was no change in expression of $\mathrm{m}^{6} \mathrm{~A}$-IncRNAs in distinct clusters. There have simply been a few studies on $\mathrm{m}^{6} \mathrm{~A}$ related IncRNA alterations. As a result, more research has responsibility for determining the precise mechanism of $\mathrm{m}^{6} \mathrm{~A}$-related IncRNA alteration and identification, as well as to confirm our findings.

FANCL (FA Complementation Group $L$ ) is a gene that codes for proteins. Fanconi Anemia, Complementation Group L, and Vacterl Association are all diseases related with FANCL. BARD1 signaling events and the BRCA1 Pathway are two of its associated pathways. ${ }^{\square}$. FANCL fold and activity may lead to carcinogenesis in these non-FA cancer patients, and the FA gene is important in the advancement of general malignancies $\llbracket$. At present, no research has been conducted on FANCL and KIRP. However, based on our findings, the aforementioned m6A-IncRNAs could be used as a therapeutic target for KIRP. 
Because there have been few studies on SETD7, further research is needed to determine the exact molecular pathways underlying the above-mentioned activity that leads to tumor suppression.

Furthermore, we investigated and computed the infiltration of various immune cells in the samples to determine the involvement of immune cell infiltration and the TME in KIRP. The invasion of Plasma cells, Macrophages M0, Macrophages M2, B cells naive, and other these cells in the TME may have a detrimental effect on the prognosis of KIRP patients, according to a research of immune cell infiltration discrepancies. Cluster 1 had a higher ESTIMATE and Stroma score, indicating that there were less tumor cell purity and greater immune-related cell infiltration in the TME. Intermediate stromal score and immune score were found to be independent risk factors for disease-free survival and overall survival in KIRP patients, with exceptional discriminatory power, accuracy, and clinical effectiveness in predicting the efficacy of KIRP genetic alterations ${ }^{\square} . M^{6}$ a and immune checkpoint inhibitors (ICls) work together to improve anticancer efficacy in ICl-resistant cancers ${ }^{\square}$. Only a small amount of research has been conducted on the relationship between $\mathrm{ICl}$ and $\mathrm{m}^{6} \mathrm{~A}$. However, we identified a substantial difference in the expression of TNFRSF4, TNFRSF9, TNFSF14, TNFRSF18, CD70, and other genes in our study. We expected that through modulating the invasion of the aforementioned immune cells, these genes could influence the prognosis of KIRP patients. Despite the fact that little is known about $\mathrm{m}^{6} \mathrm{a}$-related InRNA and KIRP. Build on the information presented above, we may conclude that immune cell infiltration of the TME affects the prognosis of KIRP patients. The lesser the tumor purity, the higher the immunological score.

In GSEA, the P53-signaling pathway was found to be the most significantly enriched pathway. p53 controls a variety of biological activities. Recent research has shown strong evidence that p53 has a role in the development of AKI and subsequent kidney healing, primarily through the regulation of apoptosis, cell cycle arrest, and autophagy ${ }^{\square}$. Furthermore, genetic and pharmacological P53 suppression has been demonstrated to affect kidney healing following AKI ${ }^{\square}$. Picking up the previously mentioned characteristics into consideration, m6A-IncRNAs may govern KIRP cell migration and proliferation through regulating the P53 SIGNALING PATHWAY. The low-risk subtype exhibited a greater survival rate than the high-risk subtype in both the test and training groups. The KIRP result can be predicted using the m6A-IncRNArelated prognostic model. In addition, our model is under a high level of accuracy in predicting KIRP patient survival. An increase in the risk score is associated with an increase in the number of death and the high-risk ratio. Our model was also unaffected by other clinical prognostic variables that have an impact on patient outcomes. As a consequence, the model might be used in a variety of clinical circumstances. As a result, our findings and the data in the literature suggested that m6A-IncRNAs could be valuable biomarkers for predicting the fate of KIRP patients.

Despite the fact that our research presents some theoretical foundations and research suggestions, there are still definite limits. To begin, we used the TCGA dataset to construct and validate a predictive signature for $\mathrm{m}^{6} \mathrm{~A}$-related IncRNAs. We were unable to get sufficient external data from other public sources to assess the model's trustworthiness. Second, we only performed preliminary expression 
analyses on the signature's $3 \mathrm{~m}^{6} \mathrm{~A}$-related IncRNAs. Nevertheless, no further functional analysis or mechanistic investigations was conducted. Finally, no experiment was conducted in KIRP to confirm the interaction between prognostic IncRNAs and $\mathrm{m}^{6} \mathrm{~A}$ modulators. However, in order to further understand the above findings, we will do additional research.

\section{Conclusions}

In summary, we assessed the expression profiles and clinical data of KIRP samples from the TCGA database to investigate prognosis-related $\mathrm{m}^{6} \mathrm{~A}$-IncRNAs. Three of the $32 \mathrm{~m}^{6} \mathrm{~A}$-related prognostic IncRNAs found in $298 \mathrm{KIRP}$ patients were included into the $\mathrm{m}^{6} \mathrm{~A}$ regulation (LUCAT1, FOXD2-AS1, and AC099850.4). For KIRP, it has a high prognostic value. Furthermore, we discovered that FANCL strongly links to overall survival. The function of prognostic FANCL was further defined through expression analysis and correlation analysis. Our findings add to our knowledge of $\mathrm{m}^{6} \mathrm{~A}$-related IncRNAs and immune cell infiltration in the TME, which could lead to different treatment targets and prognosis-related biomarkers in the future. It is desirable for our findings will aid in the identification of $\mathrm{m}^{6} \mathrm{~A}$-related IncRNA that promotes KIRP growth, so that we can learn more about their possible function in the occurrence and advancement of KIRP tumors.

\section{Abbreviations}

\begin{tabular}{|llll|}
\hline KIRP & Kidney Renal papillary cell carcinoma & TCGA & The Cancer Genome Atlas \\
\hline $\mathrm{m}^{6} \mathrm{~A}$ & N6-methyladenosine & IncRNAs & long non-coding RNAs \\
\hline AUCs & areas under the curve & DCA & Decision Curve Analysis \\
\hline TME & the tumor microenvironment & FANCL & FA Complementation Group L \\
\hline ICls & immune checkpoint inhibitors & & \\
\hline
\end{tabular}

\section{Declarations}

\section{Data availability}

Patients who have provided informed consent for the use of their data have been included in the TCGA database, which is a public database. Users can freely obtain and publish appropriate articles based on the relevant data. Our study has no ethical difficulties or conflicts of interest because it is built on opensource data.

\section{Ethics approval and consent to participation}

This manuscript is not a clinical trial, hence the ethics approval and consent to participation is not applicable. 


\section{Consent for publication}

All authors have read and approved this manuscript to be considered for publication.

\section{Competing interests}

The authors declare no competing financial interests.

\section{Acknowledgements}

Thanks to professor Huang for his strict guidance on this paper, and thanks to Miss Huang and Miss Cai of support for this paper. Thanks to reviewers and editors for their sincere comments.

\section{Fund}

1. National Natural Science Foundation of China (NSFC), 82160938, "The effect of TGF- $\beta$ 1-mediated ERK and Smad signaling pathway on the anti-renal interstitial fibrosis mechanism of drug-cake moxibustion"; 2. Health And Health Commission of Yunnan Province 2020 High-level TCM Reserve Talents (Acupuncture treatment of chronic kidney disease) Incubation project (Yunwei TCM Development Development [2021] No. 1) 3. The second round of construction project of The National Traditional Chinese Medicine School Heritage Studio of the State Administration of Traditional Chinese Medicine (National Traditional Chinese Medicine Teaching Letter [2019] 62); 4. Yunnan Provincial Health Commission "2020 High-level Traditional Chinese Medicine Talents Training Target" project (Yunnan Traditional Chinese Medicine Development (2021) No. 1); 5. Yunnan Provincial Department of Science and Technology-Joint Special General Project of Traditional Chinese Medicine (No.2019FF002-022); 6. Social Science Project of Yunnan University of Traditional Chinese Medicine (No. [2020] -SkyB-00016).

\section{Author Contributions}

Zixuan Wu drafted and revised the manuscript. Xuyan Huang and Minjie Cai are in charge of data collection. Peidong Huang conceived and designed this article, in charge of syntax modification and revise of the manuscript. All the authors have read and agreed to the final version manuscript.

\section{References}

1. Znaor Ariana,Lortet-Tieulent Joannie,Laversanne Mathieu,et al. International variations and trends in renal cell carcinoma incidence and mortality.[J]. European urology,2015,67(3):

2. Rebecca L. Siegel MPH,Kimberly D. Miller MPH,Ahmedin Jemal DVM, PhD. Cancer statistics, 2019[J]. CA: A Cancer Journal for Clinicians,2019,69(1):

3. Gabriel G. Malouf,Xiaoping Su,Jianping Zhang,et al. DNA Methylation Signature Reveals Cell Ontogeny of Renal Cell Carcinomas[J]. Clinical Cancer Research,2016,22(24):

4. Linehan WM,Spellman PT,Ricketts $\mathrm{CJ}$,et al. Comprehensive Molecular Characterization of Papillary Renal-Cell Carcinoma[J]. The New England Journal of Medicine,2016,374(2): 
5. Tachibana Hidekazu,Kondo Tsunenori,Ishihara Hiroki,et al. Modest efficacy of nivolumab plus ipilimumab in patients with papillary renal cell carcinoma[J]. Japanese Journal of Clinical Oncology,2021,51(4):

6. Chen,Cheng,Li. The molecular characterization and therapeutic strategies of papillary renal cell carcinoma[J]. Expert Review of Anticancer Therapy,2019,19(2):

7. Giulia Courthod,Marcello Tucci,Massimo Di Maio,et al. Papillary renal cell carcinoma: A review of the current therapeutic landscape[J]. Critical Reviews in Oncology / Hematology,2015,96(1):

8. Eich Marie-Lisa,Chaux Alcides,Mendoza Rodriguez Maria Angélica,et al. Tumour immune microenvironment in primary and metastatic papillary renal cell carcinoma.[J]. Histopathology,2020,76(3):

9. Cao Haoyuan,Zhang Jiandong,Wang Wei. DAB2IP Plays Important Clinical Significance and Correlates With Immune Infiltration in Renal Cell Carcinoma.[J]. Technology in cancer research \& treatment,2020,19:

10. Liu Nian,Pan Tao. $\mathrm{N}^{6}$-methyladenosine-encoded epitranscriptomics.[J] .Nat Struct Mol Biol, 2016, 23 : 98-102.

11. Boxuan Simen Zhao,lan A. Roundtree,Chuan He. Post-transcriptional gene regulation by mRNA modifications[J]. Nature Reviews Molecular Cell Biology,2017,18(1):

12. Yu JunJie,Mao WeiPu,Sun Si,et al. Identification of an $\mathrm{m}^{6} \mathrm{~A}-$ Related IncRNA Signature for Predicting the Prognosis in Patients With Kidney Renal Clear Cell Carcinoma [J]. Frontiers in Oncology,2021:

13. Sara Zaccara,Ryan J. Ries,Samie R. Jaffrey. Reading, writing and erasing mRNA methylation[J]. Nature Reviews Molecular Cell Biology,2019,20(13):

14. Zheng Jianfeng,Guo Jialu,Cao Benben,et al. Identification and validation of IncRNAs involved in $\mathrm{m}^{6} \mathrm{~A}$ regulation for patients with ovarian cancer.[J]. Cancer cell international,2021,21(1):

15. Wang Tianyi,Kong Shan,Tao Mei,et al. The potential role of RNA N6-methyladenosine in Cancer progression.[J]. Molecular cancer,2020,19(1):

16. Jin-zhao Ma,Fu Yang,Chuan-chuan Zhou,et al. METTL14 suppresses the metastatic potential of hepatocellular carcinoma by modulating N6-methyladenosine-dependent primary MicroRNA processing[J]. Hepatology,2017,65(2):

17. Shaoyun Wang,Peiwei Chai,Ruobing Jia,et al. Novel insights on $\mathrm{m}^{6} \mathrm{~A}$ RNA methylation in tumorigenesis: a double-edged sword[J]. BioMed Central,2018,17(1):

18. He Liujia,Li Jiangfeng,Wang Xiao,et al. The dual role of N6-methyladenosine modification of RNAs is involved in human cancers.[J]. Journal of cellular and molecular medicine,2018,22(10):

19. Evans Joseph R,Feng Felix Y,Chinnaiyan Arul M. The bright side of dark matter: IncRNAs in cancer.[J]. The Journal of clinical investigation,2016,126(8):

20. Adam M. Schmitt,Howard Y. Chang. Long Noncoding RNAs in Cancer Pathways[J]. Cancer Cell,2016,29(4): 
21. Zhao Enfa,Chen Shimin,Dang Ying. Development and External Validation of a Novel Immune Checkpoint-Related Gene Signature for Prediction of Overall Survival in Hepatocellular Carcinoma.[J]. Frontiers in molecular biosciences,2020,7:

22. Wang Zhining,Jensen Mark A,Zenklusen Jean Claude. A Practical Guide to The Cancer Genome Atlas (TCGA).[J]. Methods in molecular biology (Clifton, N.J.),2016,1418:

23. UniProt: the universal protein knowledgebase. Nucleic Acids Res. 45, D158-D169. https ://doi.org/10.1093/nar/gkw1099 (2017).

24. Xie Yuan-Hong,Chen Ying-Xuan,Fang Jing-Yuan. Comprehensive review of targeted therapy for colorectal cancer.[J]. Signal transduction and targeted therapy,2020,5(1):

25. Sara Zaccara,Ryan J. Ries,Samie R. Jaffrey. Reading, writing and erasing mRNA methylation[J]. Nature Reviews Molecular Cell Biology,2019,20(13):

26. Yuying Han,Qiyu Zheng,Ye Tian,et al. Identification of a nine-gene panel as a prognostic indicator for recurrence with muscle-invasive bladder cancer[J]. Journal of Surgical Oncology,2019,119(8):

27. Dai Dongjun,Wang Hanying,Zhu Liyuan,et al. $\mathrm{N}^{6}$-methyladenosine links RNA metabolism to cancer progression.[J]. Cell death \& disease,2018,9(2):

28. Ma Shuai,Chen Chen,Ji Xiang,et al. The interplay between $\mathrm{m}^{6} \mathrm{~A}$ RNA methylation and noncoding RNA in cancer.[J]. Journal of hematology \& oncology,2019,12(1):

29. Zhou Qiwei,Li Diangeng,Zheng Hongying,et al. A novel IncRNA-miRNA-mRNA competing endogenous RNA regulatory network in lung adenocarcinoma and kidney renal papillary cell carcinoma.[J]. Thoracic cancer,2021:

30. He Ruyi,Wang Longyu,Li Juan,et al. Integrated Analysis of a Competing Endogenous RNA Network Reveals a Prognostic Signature in Kidney Renal Papillary Cell Carcinoma[J]. Frontiers in Cell and Developmental Biology,2020:

31. Liu Nian,Zhou Katherine I,Parisien Marc,et al. $\mathrm{N}^{6}$-methyladenosine alters RNA structure to regulate binding of a low-complexity protein.[J]. Nucleic acids research,2017,45(10):

32. [1]Yajuan Yang,Ting Guo,Ran Liu,Hanni Ke,Weiwei Xu,Shidou Zhao,Yingying Qin. FANCL gene mutations in premature ovarian insufficiency[J]. Human Mutation,2020,41(5):

33. [1]Frost Mark G,Mazloumi Aboukheili Amir Mahdi,Toth Rachel,Walden Helen. Characterization of FANCL variants observed in patient cancer cells.[J]. Bioscience reports,2020,40(6):

34. Yu Zhong Lin,Zhu Zheng Ming. Comprehensive analysis of $\mathrm{N}^{6}$-methyladenosine -related long noncoding RNAs and immune cell infiltration in hepatocellular carcinoma.[J]. Bioengineered,2021,12(1):

35. Wang Yi,Li Na,Tian Di,et al. Analysis of m6A-Related IncRNAs for Prognosis Value and Response to Immune Checkpoint Inhibitors Therapy in Hepatocellular Carcinoma.[J]. Cancer management and research,2021,13:

36. Marianne Farnebo,Vladimir J.N. Bykov,Klas G. Wiman. The p53 tumor suppressor: A master regulator of diverse cellular processes and therapeutic target in cancer[J]. Biochemical and Biophysical Research Communications,2010,396(1): 
37. Tang Chengyuan,Ma Zhengwei,Zhu Jiefu,et al. P53 in kidney injury and repair: Mechanism and therapeutic potentials.[J]. Pharmacology \& therapeutics,2019,195:

\section{Figures}

(a)

$$
\text { - IncRNA - m6A }
$$

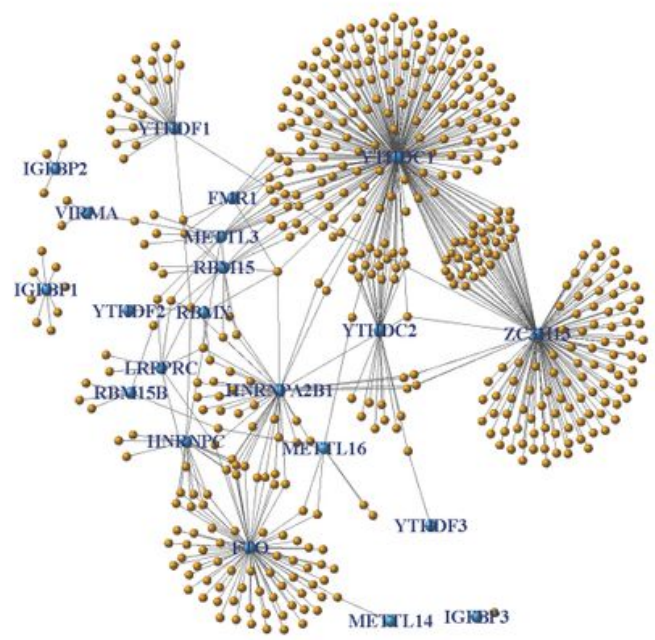

(c)

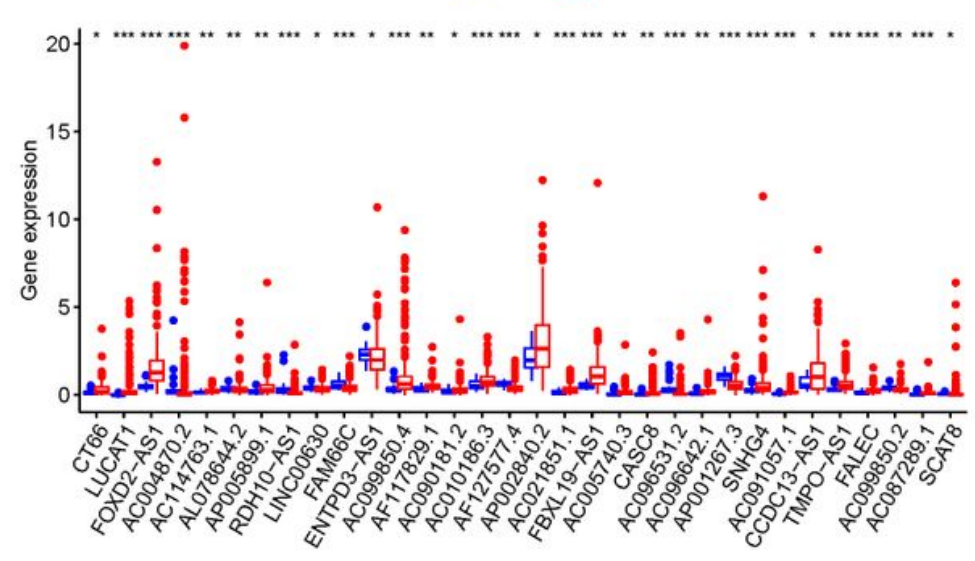

(b)

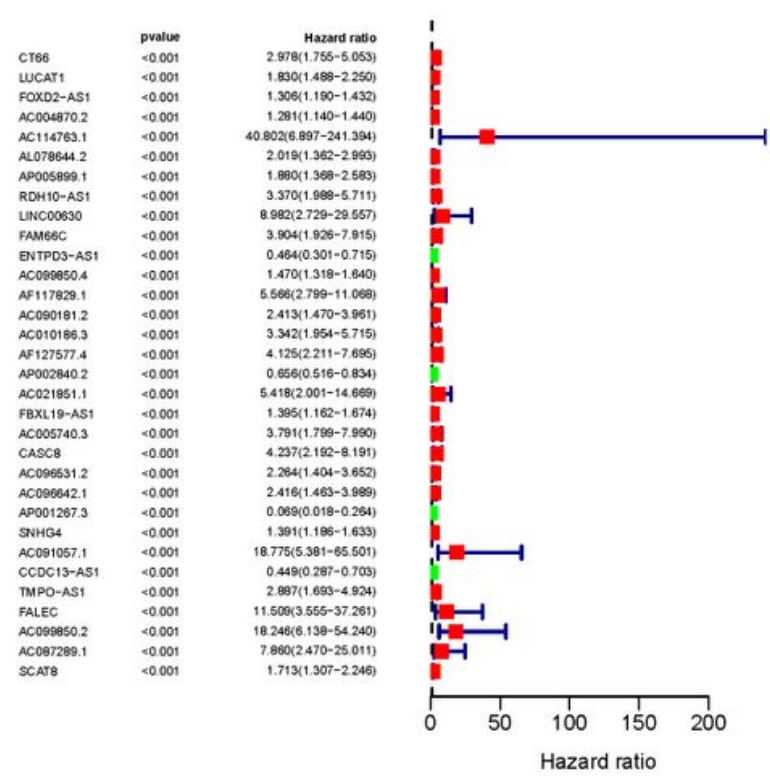

(d)

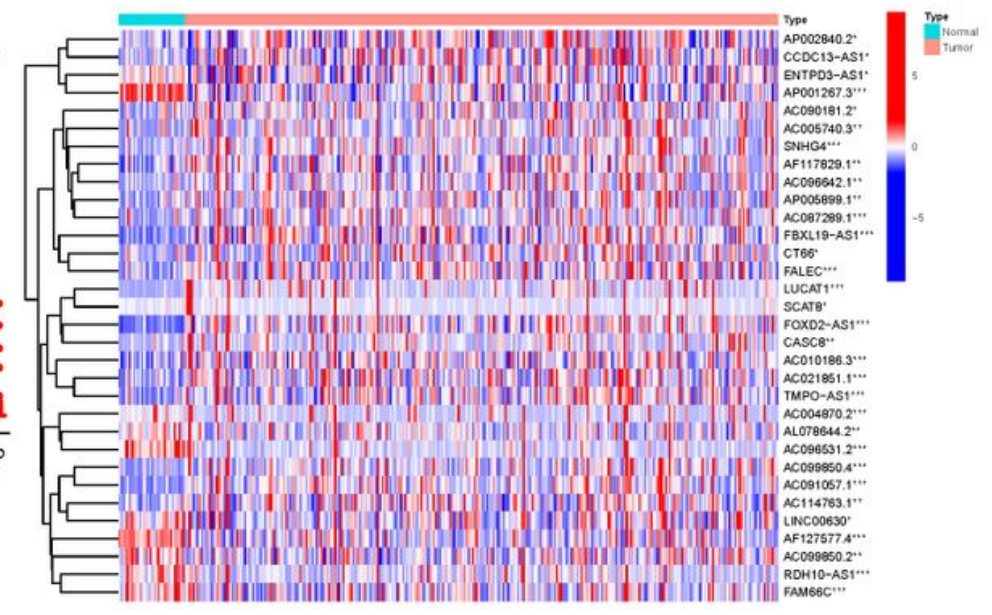

\section{Figure 1}

The function of m6A-IncRNAs and their role in the prognosis of patients with KIRP. (a): Correlation between m6A-related gene and IncRNAs. (b): Univariate Cox regression analysis forest plot. (c): Boxplot showing changes in m6A-prognostic IncRNA expression. (d): Heatmap. 
(a)

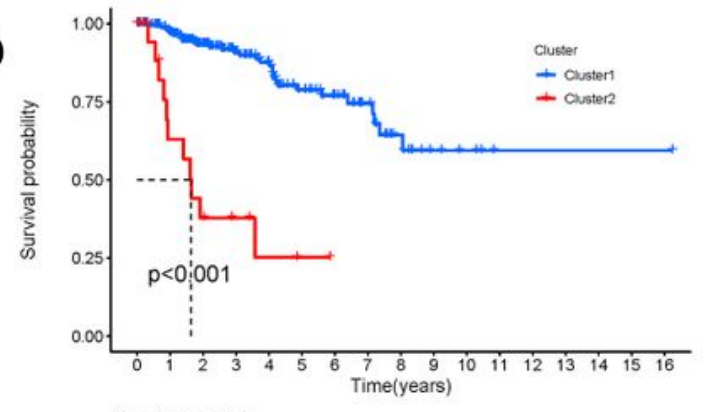

Number at risk

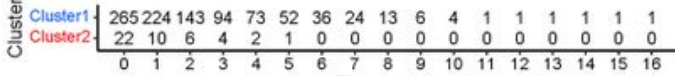

Cluster 间 Cluster1 由 Cluster2

(c)

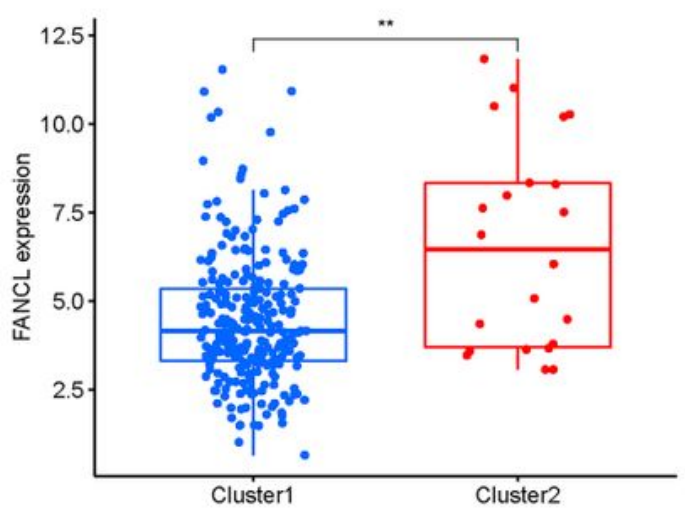

(b)

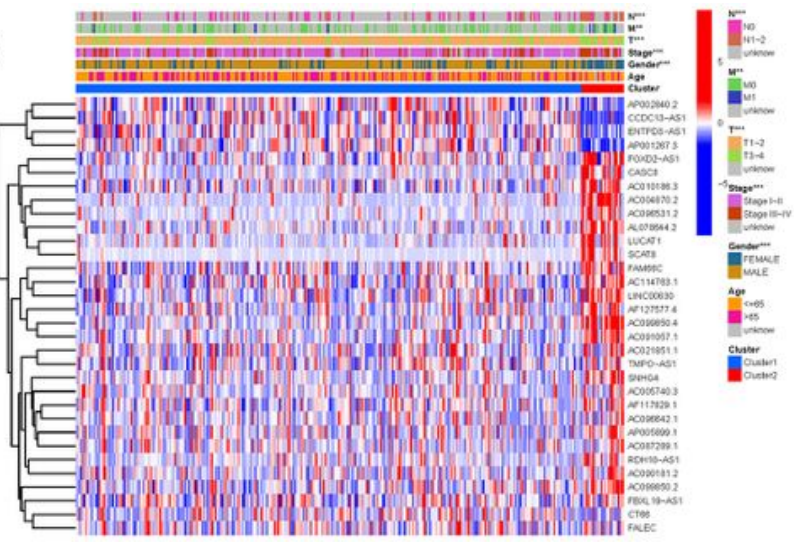

Type 审 Normal 审 Tumor

(d)

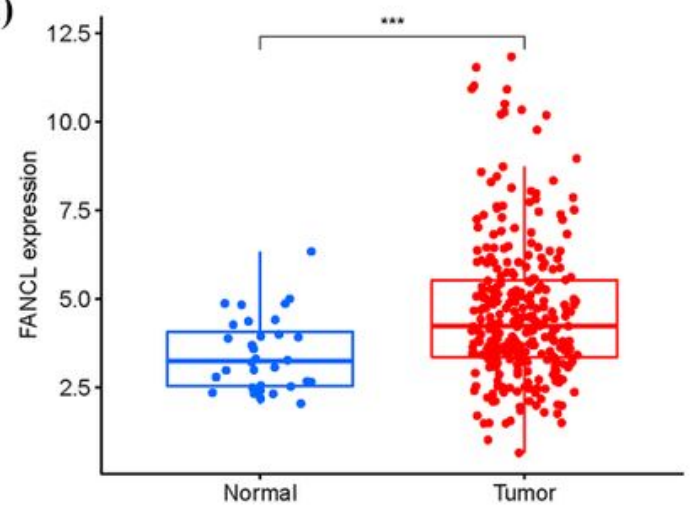

(e)

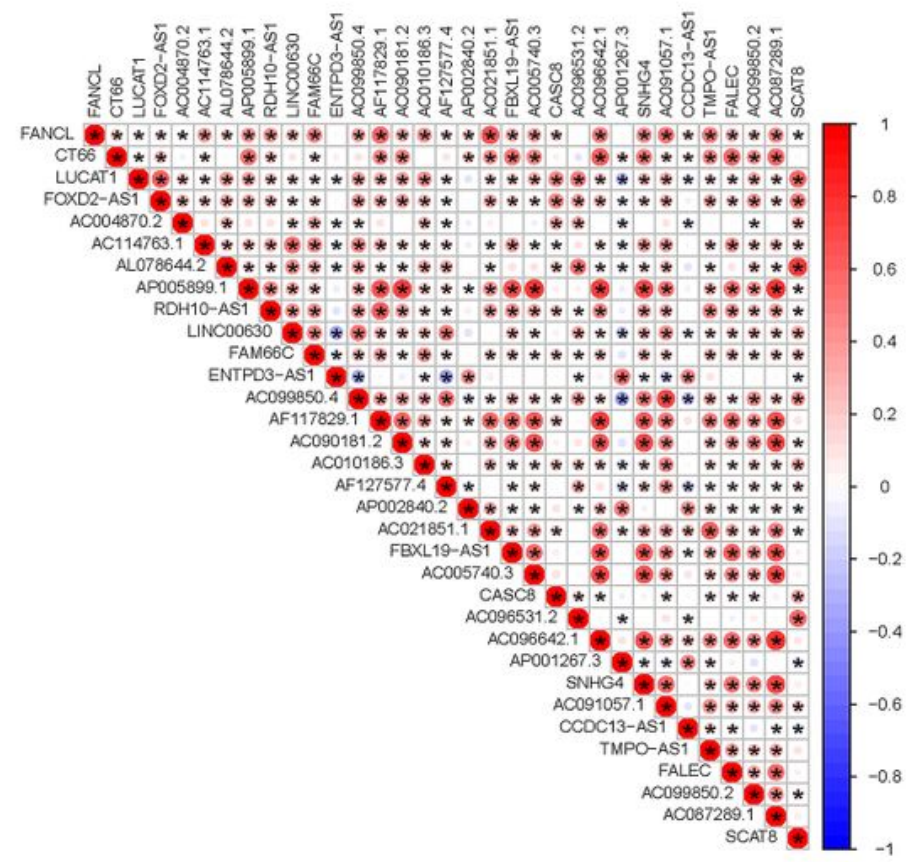

\section{Figure 2}

The expression and relationship of m6A prognostic IncRNAs and target gene.(a): Survival analysis. (b + c): Differences in the expression. (d): Heatmap. (e): Correlation analysis. 

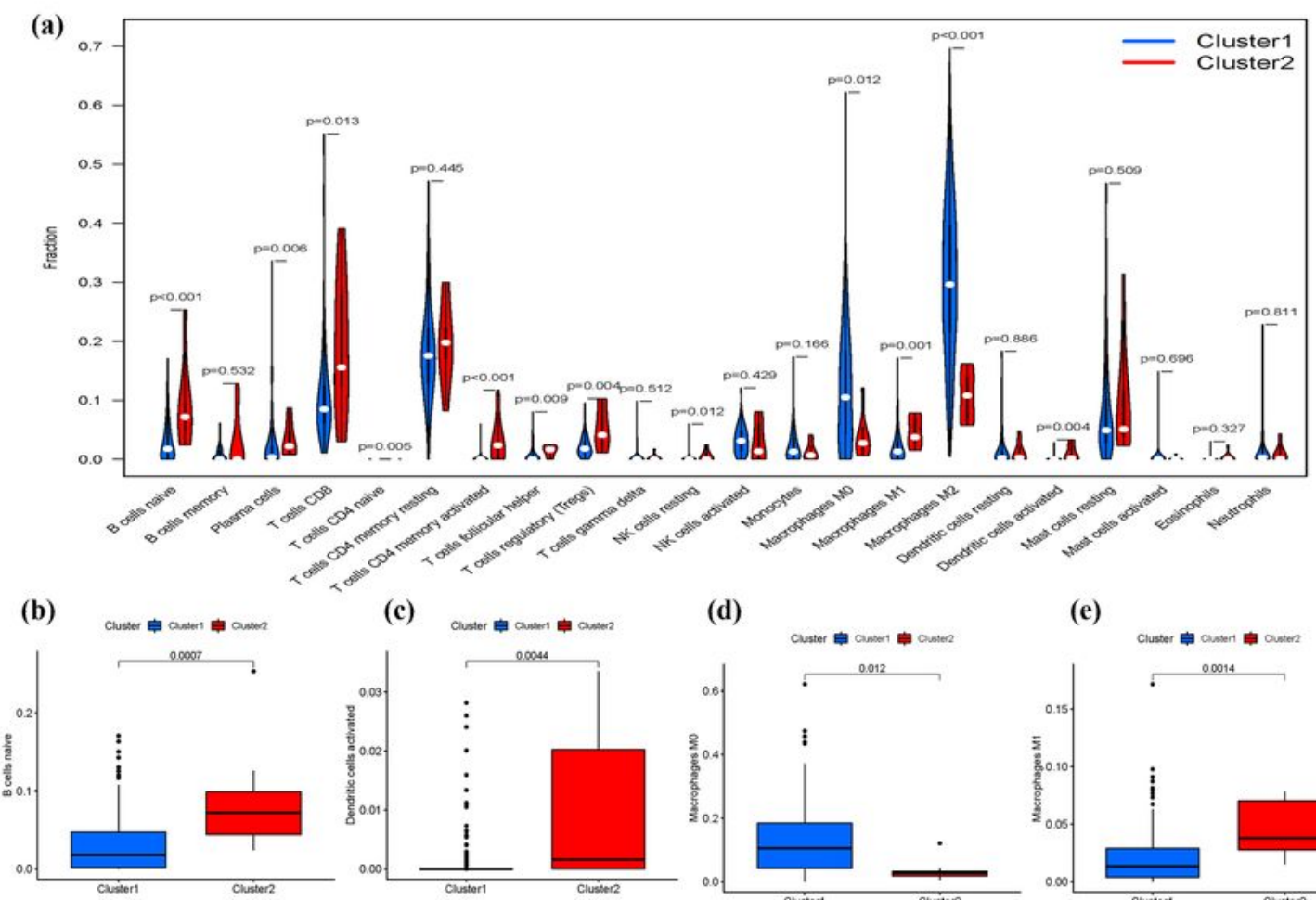

(d)

(e)
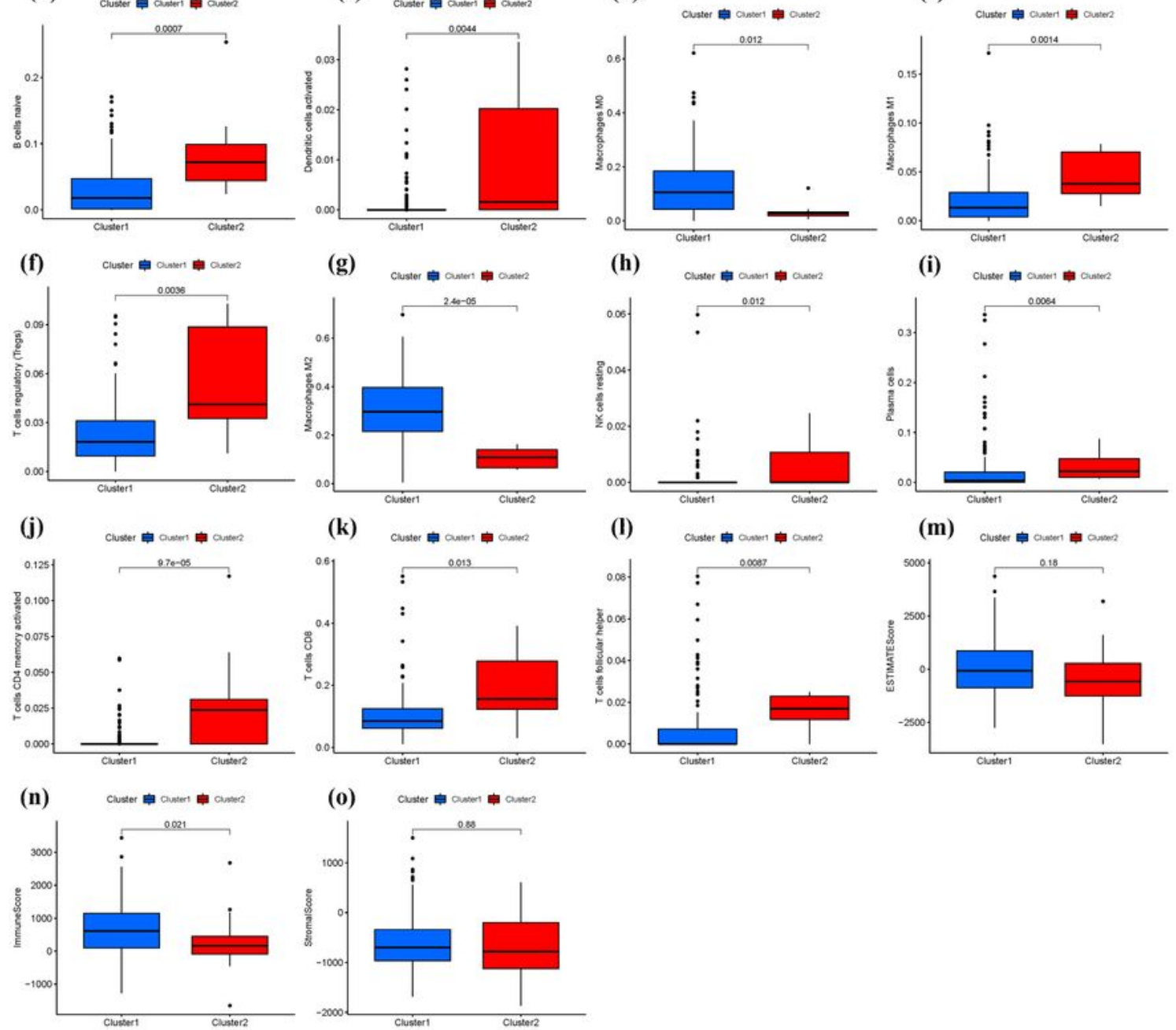

Figure 3

Analysis of immune cell infiltration and the tumor microenvironment. (a) Vioplot. (b-i) Boxplot. (m-o): Differential analysis. 

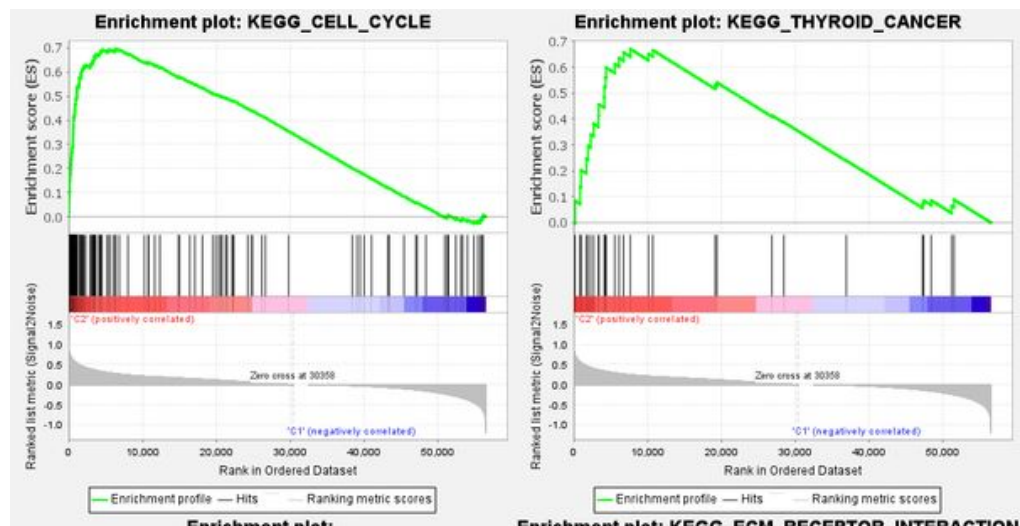

Enrichment plot: KEGG_DNA_REPLICATION

KEGG_GLYCOSAMINOGLYCAN_BIOSYNTHESIS_KERAT AN_SULFATE

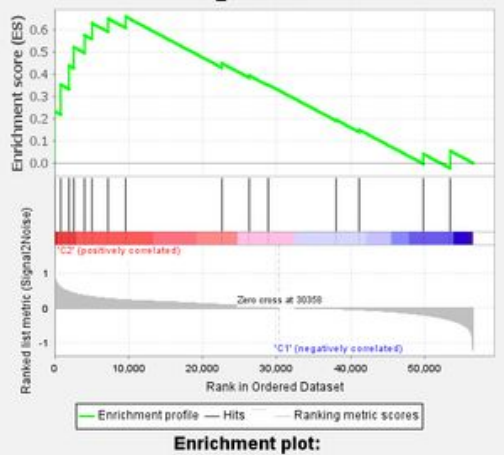

Enrichment plot: KEGG_ECM_RECEPTOR_INTERACTION Enrichment plot: KEGG_P53_SIGNALING_PATHWAY
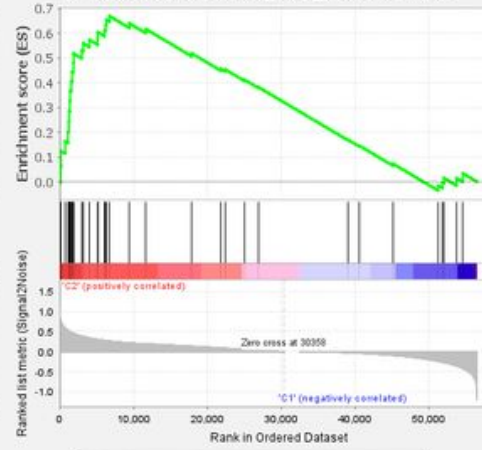

-Enichments proflla - Hits - Raning metric scores
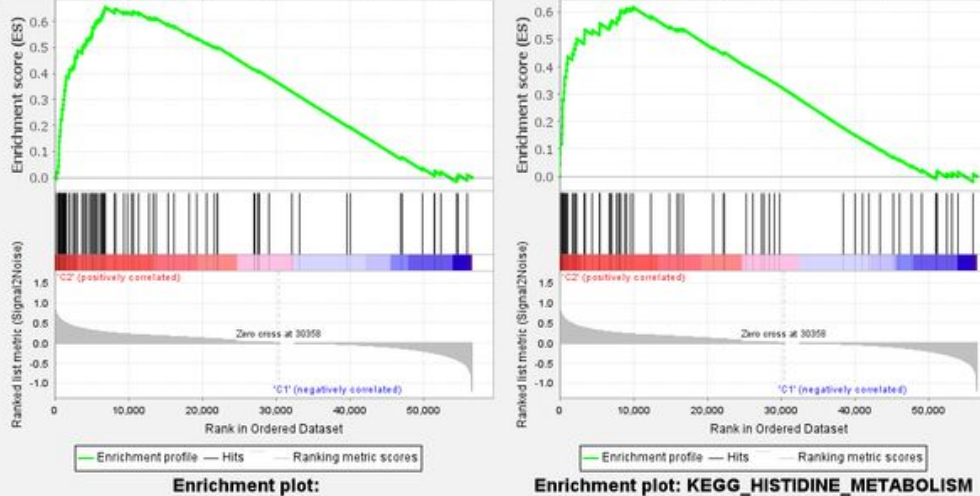

KEGG_VALINE_LEUCINE_AND_ISOLEUCINE_DEGRADA KEGG_OXIDATIVE_PHOSPHORYLATION
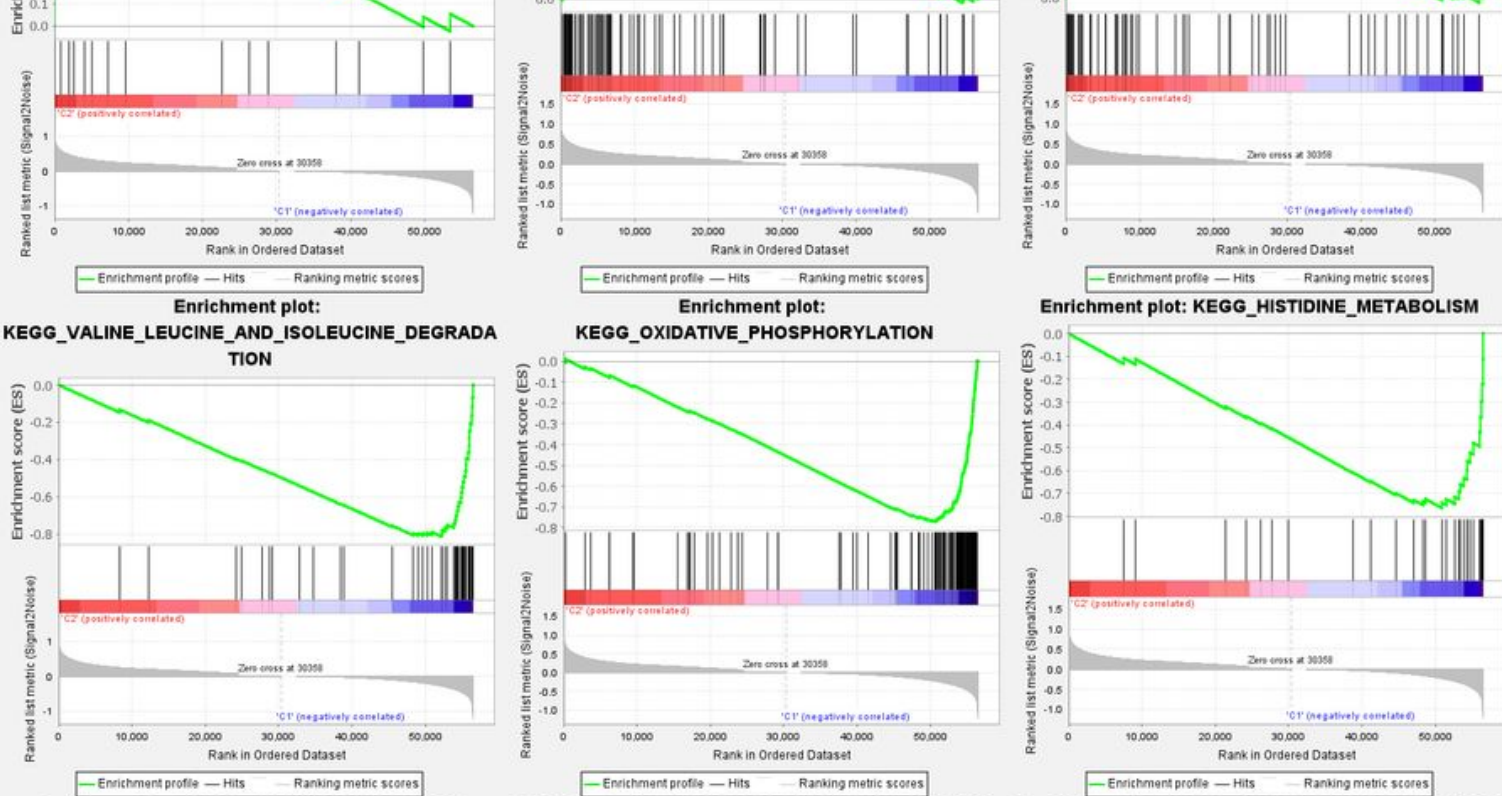

hrichment plot: KEGG_PARKINSONS_DISEASE
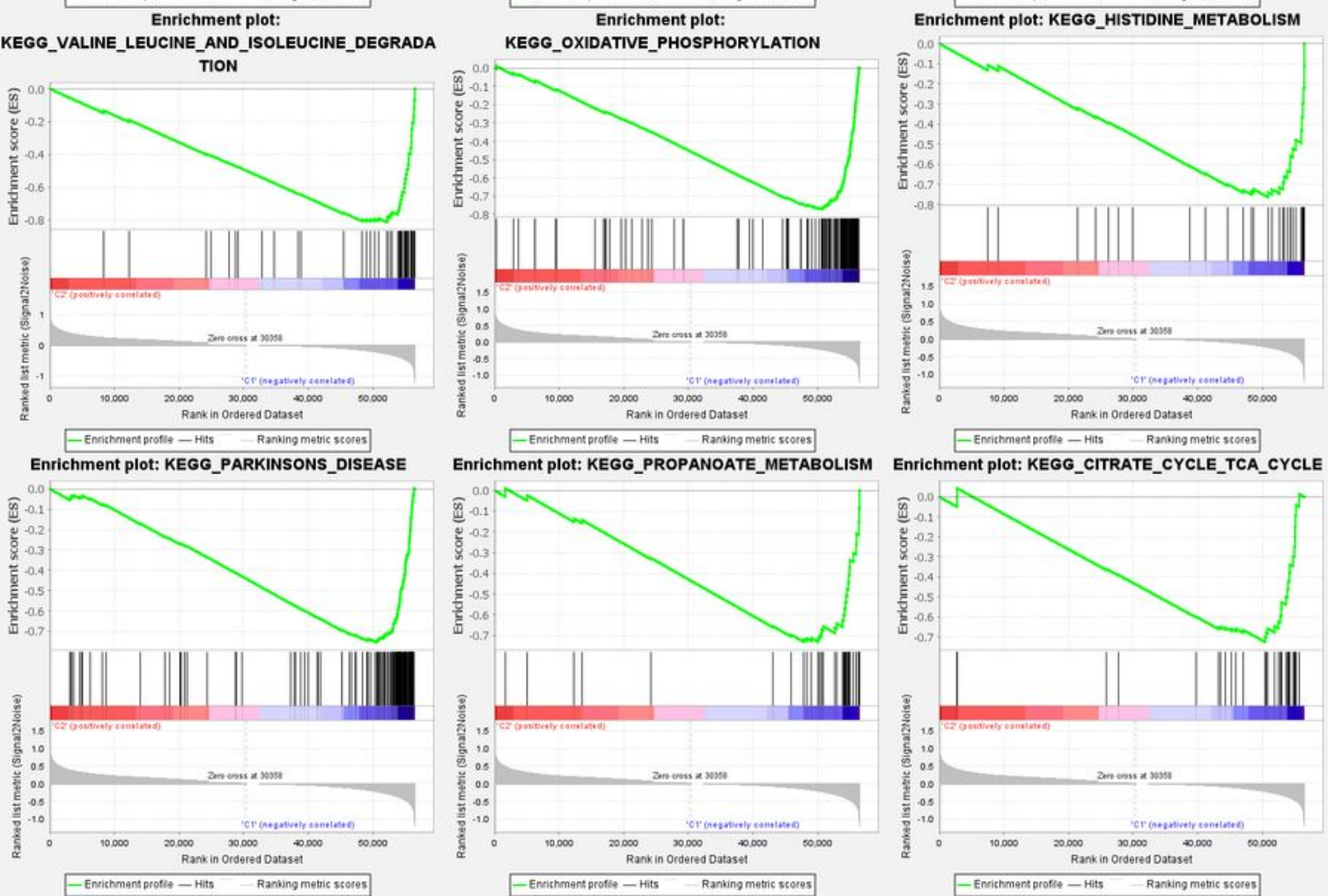

Figure 4

Gene set enrichment analysis. 


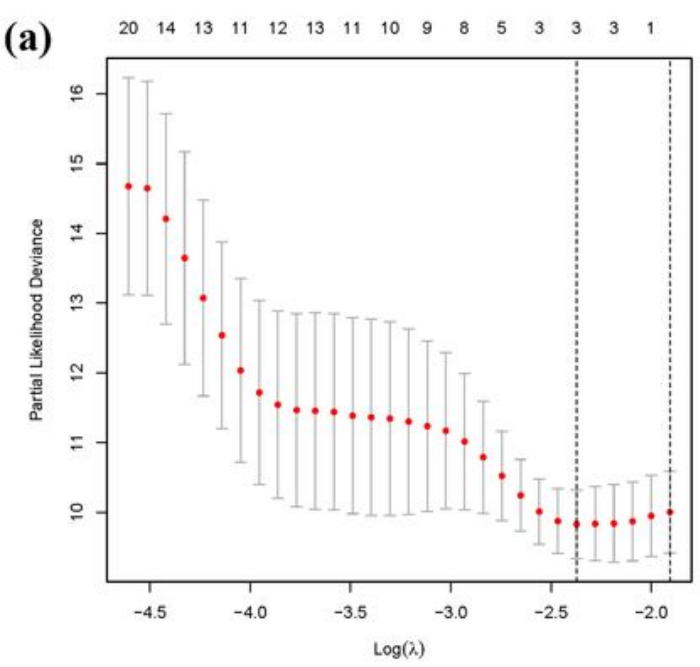

(c)

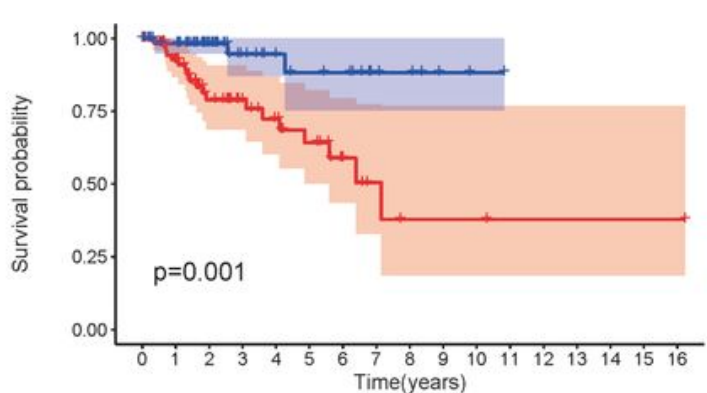

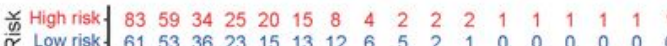

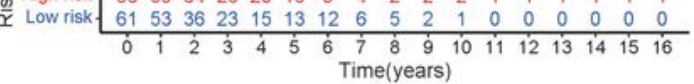

(e)

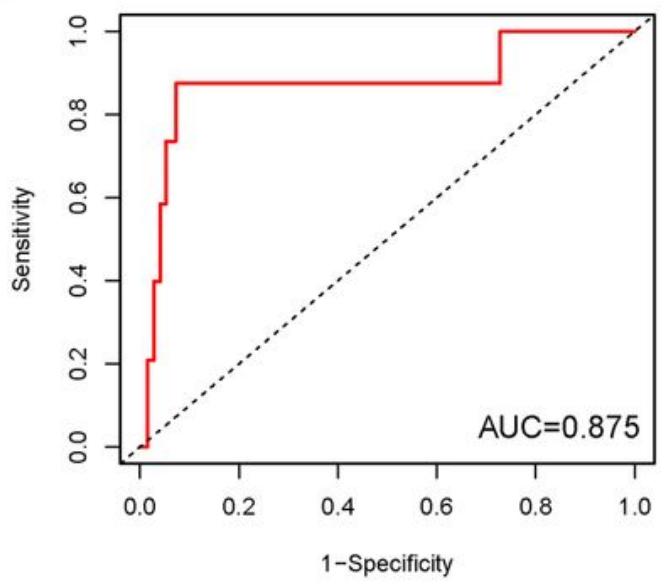

(b)

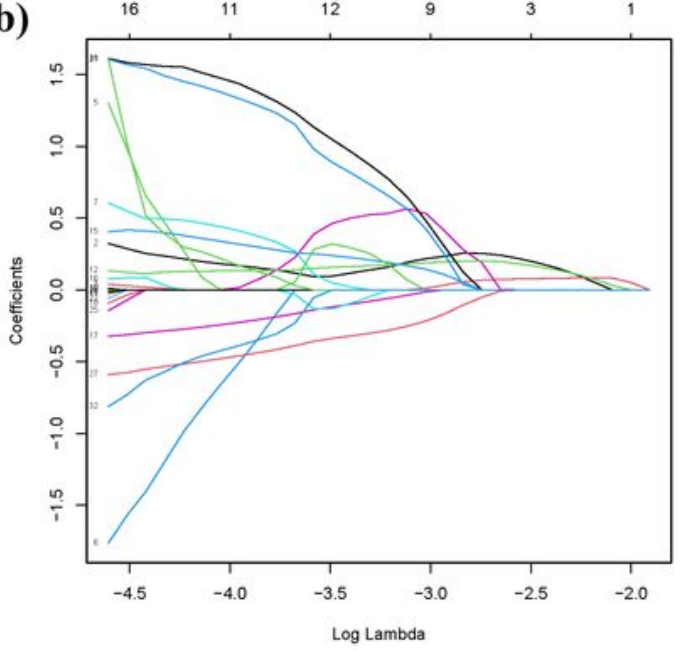

(d)

Risk + High nisk $\perp$ Low risk
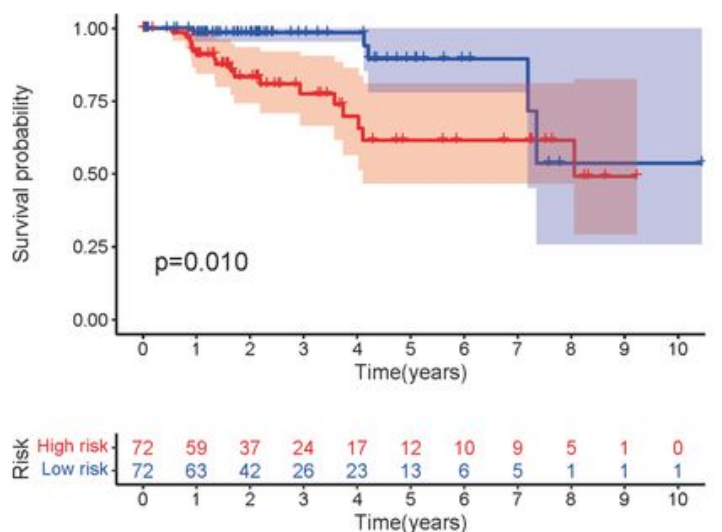

(f)

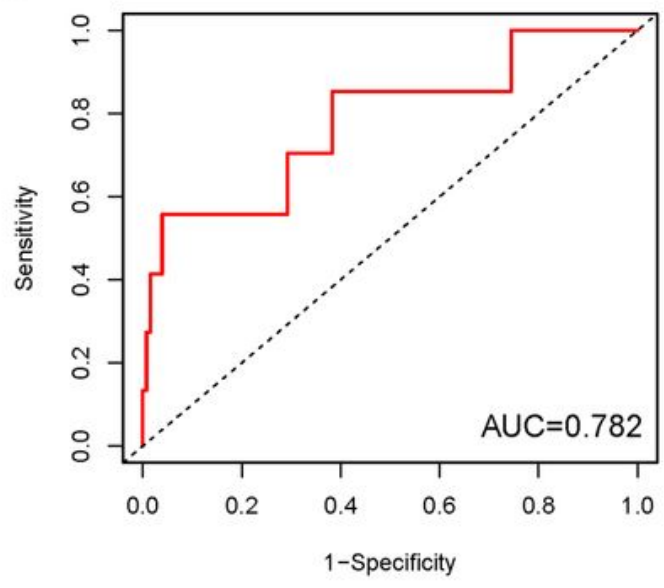

Figure 5

Prognostic model and its impact on KIRP patients' prognosis. $(a+b)$ : A lasso regression model was used to construct a prognostic model. $(c+d)$ Different groups' survival curves. $(e+f)$ : Receiver operating characteristics curve. 


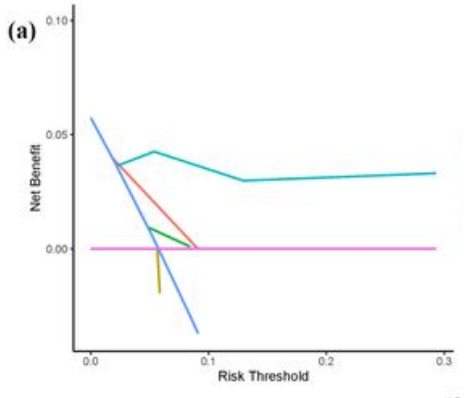

(e)

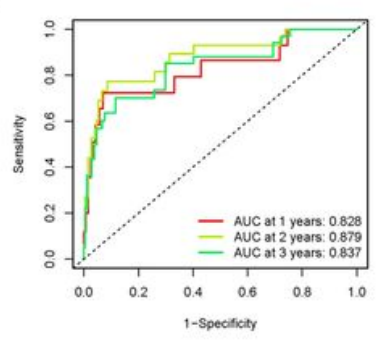

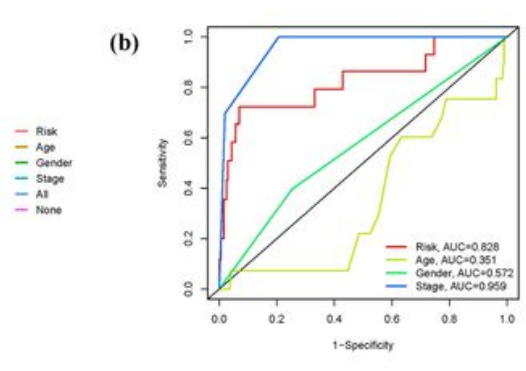

(c)
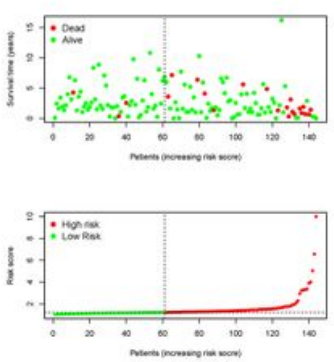

(f)

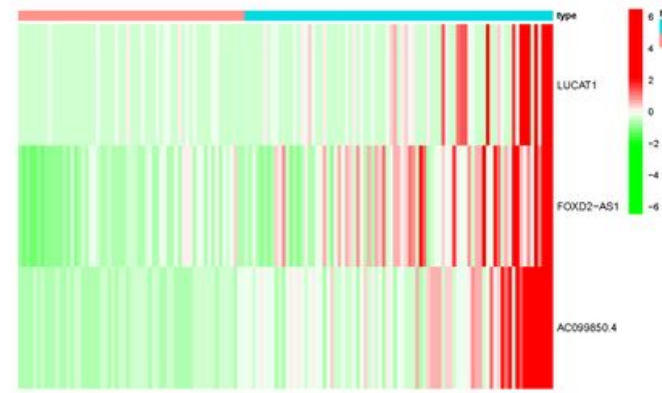

(d)
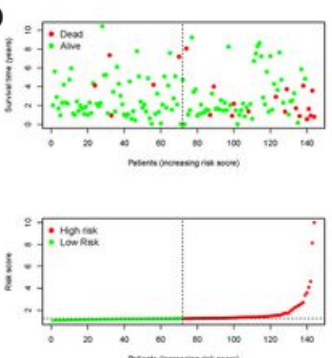

Figure 6

m6A-IncRNAs signature. (a) The AUC values. (b). The DCA. (c-d). Risk survival status plot, (e). The AUC of the for the prediction of 1, 2, 3-year survival rate. $(f-g)$ Heatmap. $(c+f)$ : test group. $(d+g)$ : training group.

(a)

$\begin{array}{lrr} & \text { pvalue } & \text { Hazard ratio } \\ & & \\ \text { Age } & 0.952 & 1.001(0.965-1.039) \\ \text { Gender } & 0.613 & 0.740(0.231-2.376) \\ & & \\ \text { Stage } & 0.006 & 1.930(1.212-3.074) \\ & & \\ \text { riskScore } & <0.001 & 2.973(1.706-5.183)\end{array}$

(c)

$\begin{array}{lrr} & \text { pvalue } & \text { Hazard ratio } \\ \text { Age } & 0.712 & 0.993(0.955-1.032) \\ \text { Gender } & 0.666 & 1.278(0.419-3.895) \\ & & \\ \text { Stage } & <0.001 & 2.424(1.628-3.609) \\ & & \\ \text { riskScore } & <0.001 & 4.197(2.557-6.890)\end{array}$

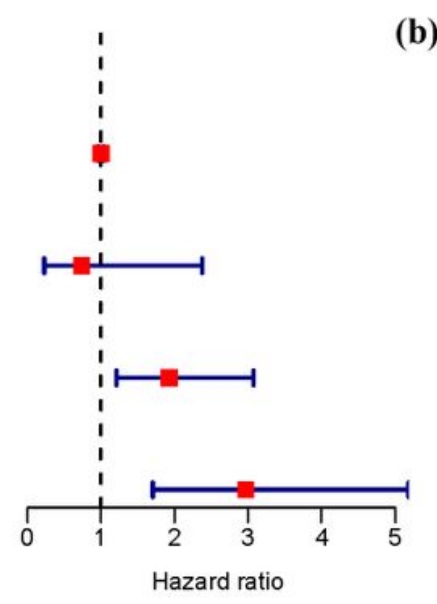

(d)

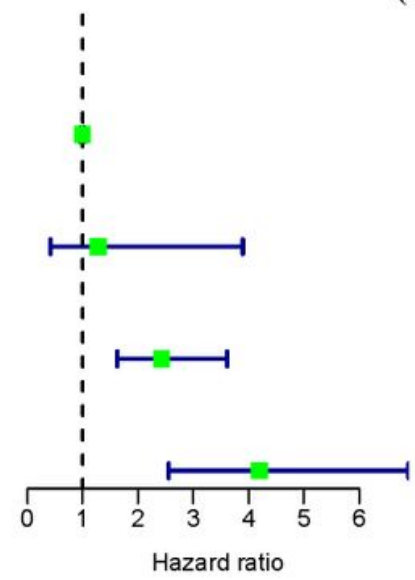

(b)
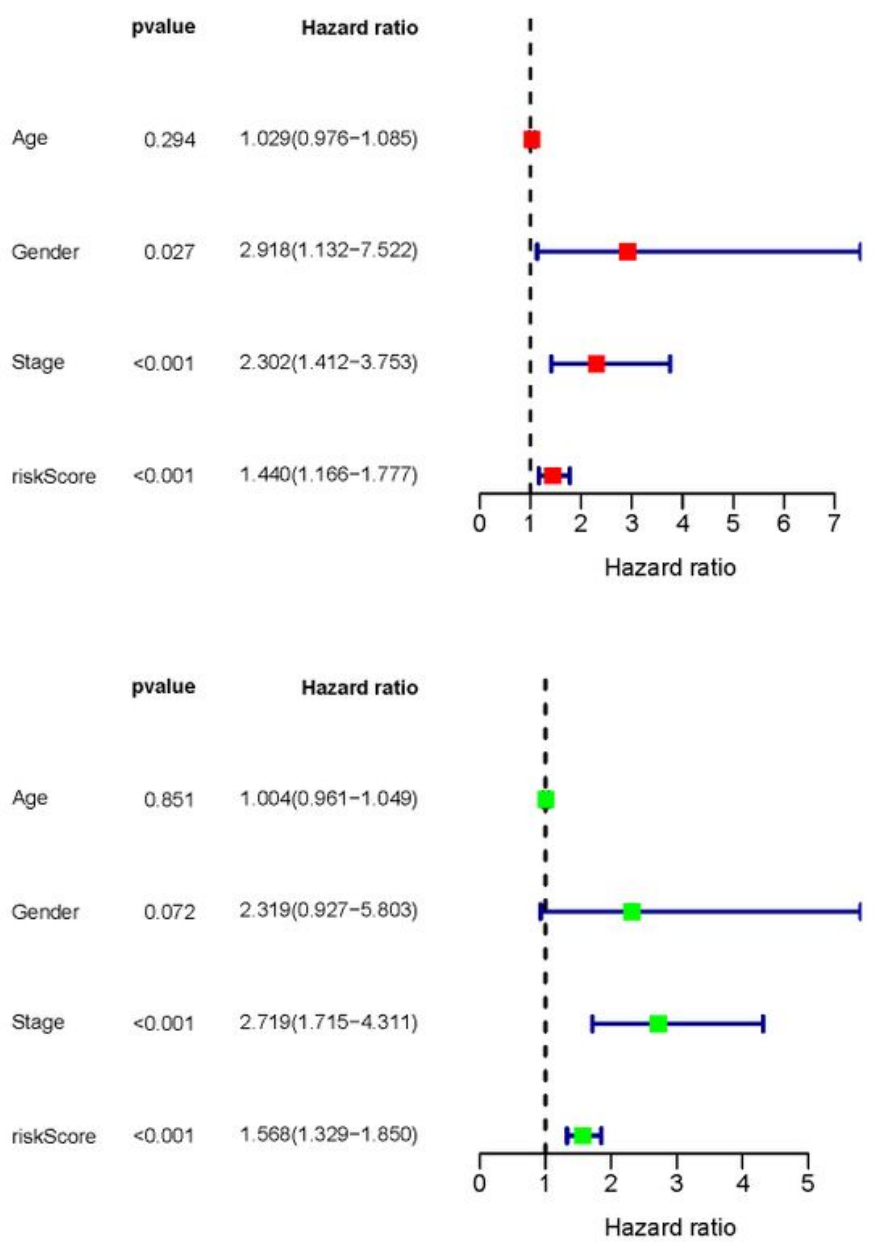
Figure 7

Univariate analyses of independent prognostic analysis. $(a+c)$ : test group, $(b+d)$ : training group.

Patients with age $>65$

Risk + high + low

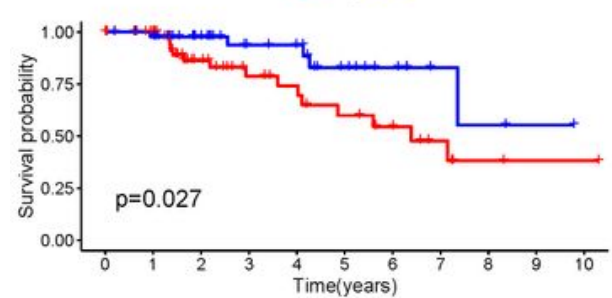

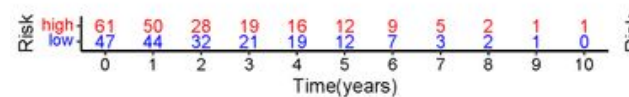
Patients with MALE

Risk - high - low

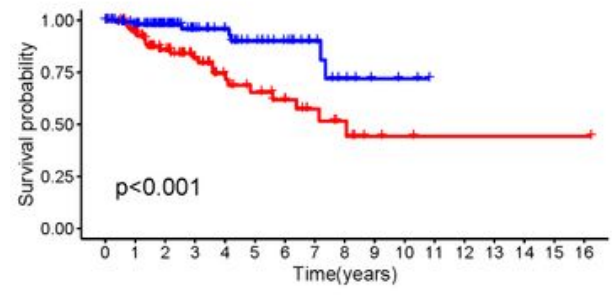

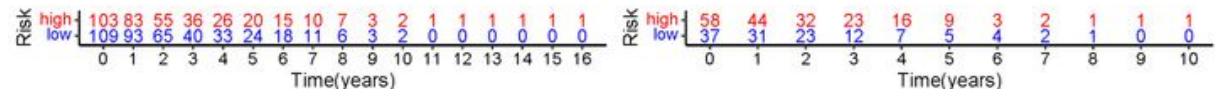

Patients with NO

Risk - high - low

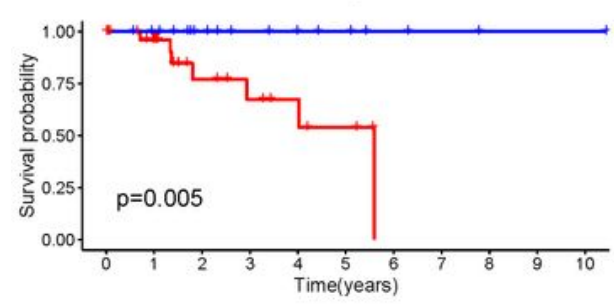

尊 high. \begin{tabular}{ccccccccccc}
31 & 21 & 10 & 7 & 5 & 3 & 0 & 0 & 0 & 0 & 0 \\
18 & 16 & 11 & 8 & 6 & 5 & 3 & 2 & 1 & 1 & 1 \\
\hline 0 & 1 & 2 & 3 & 4 & 5 & 6 & 7 & 8 & 9 & 10 \\
& & & &
\end{tabular} Patients with Stage III-IV

Risk - high - low

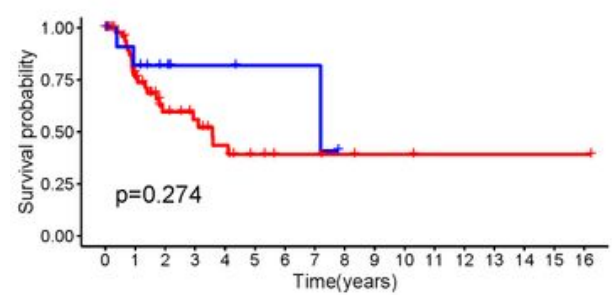

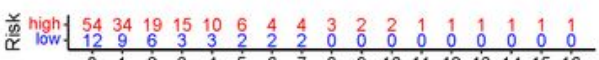
$\begin{array}{lllllllllll}6 & 7 & 8 & 9 & 10 & 11 & 12 & 13 & 14 & 15 & 16 \\ \text { Time(years) }\end{array}$
Risk + high - low

Time(years)
Patients with FEMALE

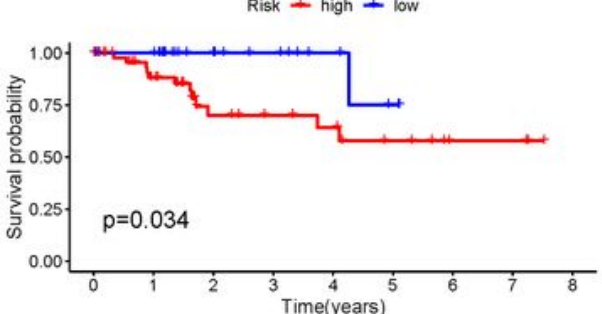

Patients with M1

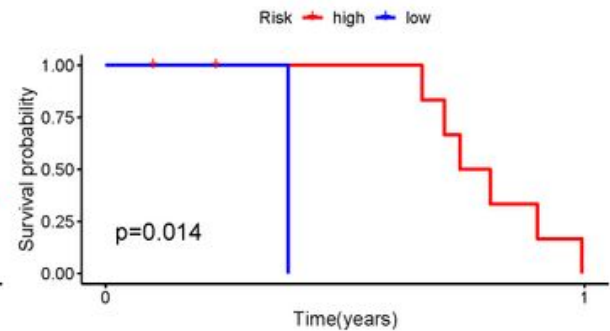

Patients with Stage I-II

Risk - high - low

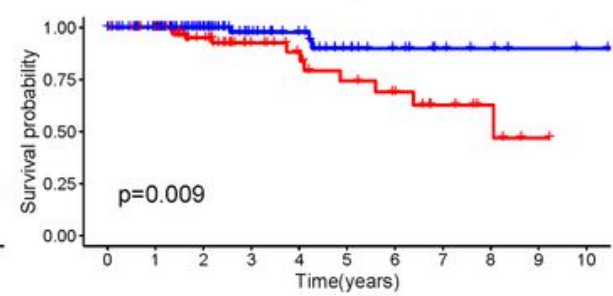

Risk + high + low

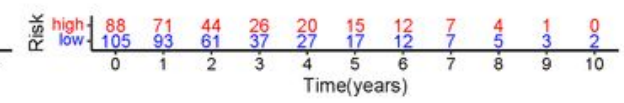

Patients with T3-4

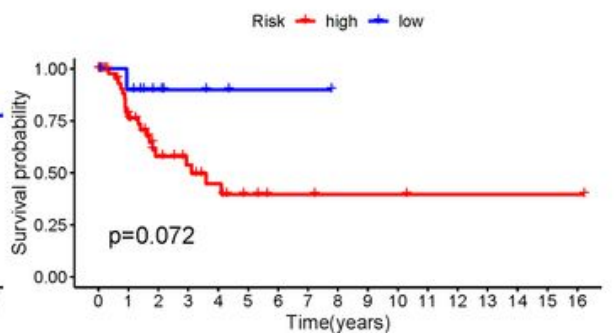

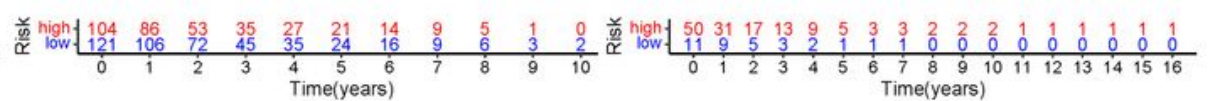

\section{Figure 8}

Survival curve for model validation. age $(a+b)$, sex $(c+d)$, M stage $(e+f), N$ stage $(g+h)$, stage $(i+j)$, and $T$ stage $(k+l)$. 


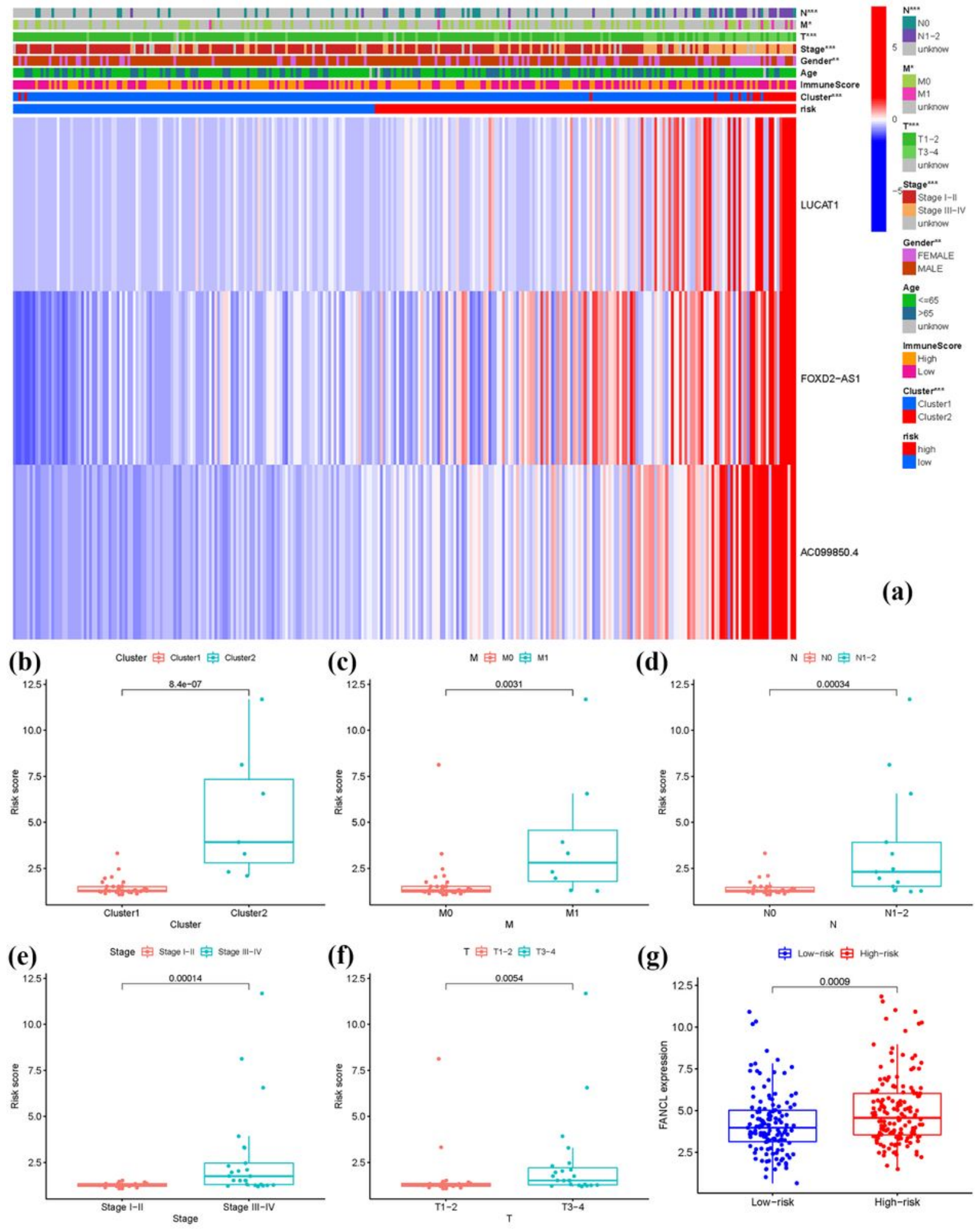

Figure 9

Correlation analysis of risk, clinical and genetic differences analysis of the target gene. (a): Heatmap of risk and clinical correlation analysis; (b-f): Boxplot of risk and clinical correlation analysis. (g): Analysis of genetic differences in target gene. 


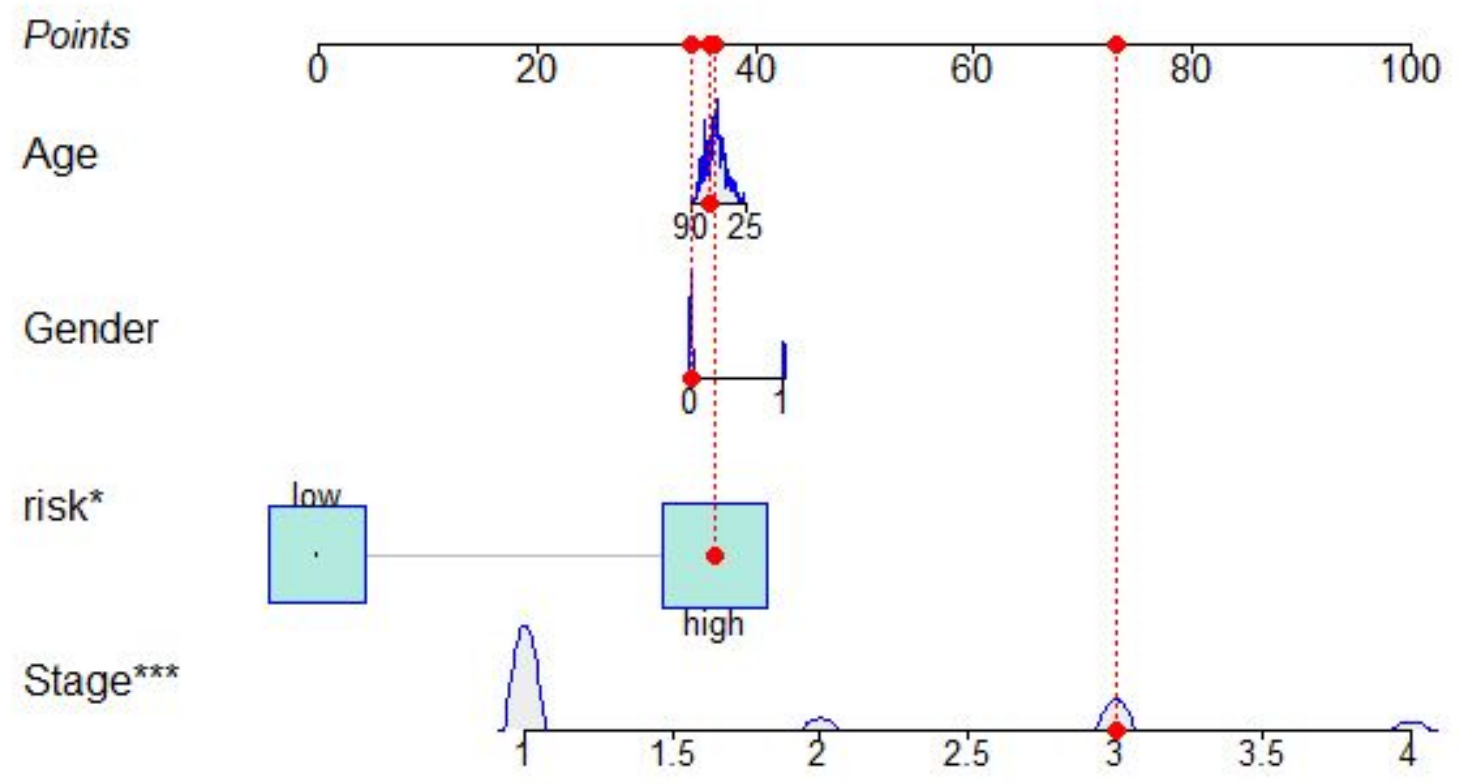

\section{Total points}

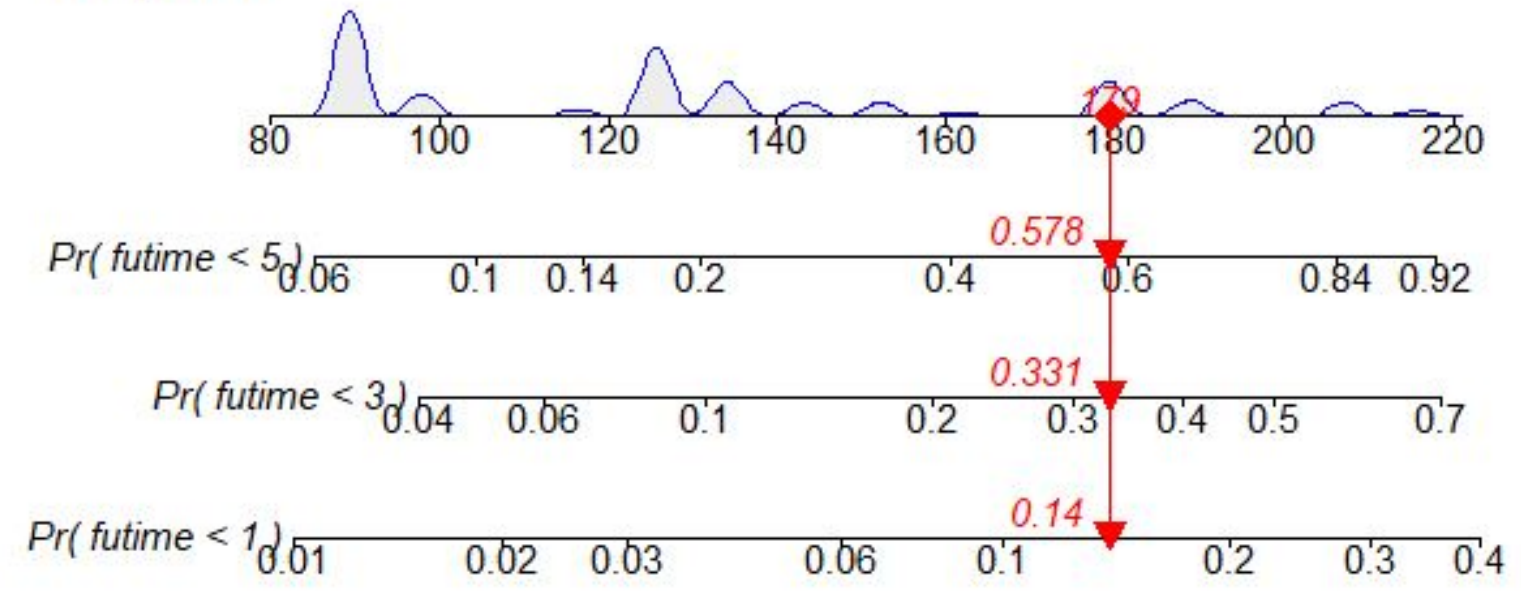

Figure 10

A nomogram. 

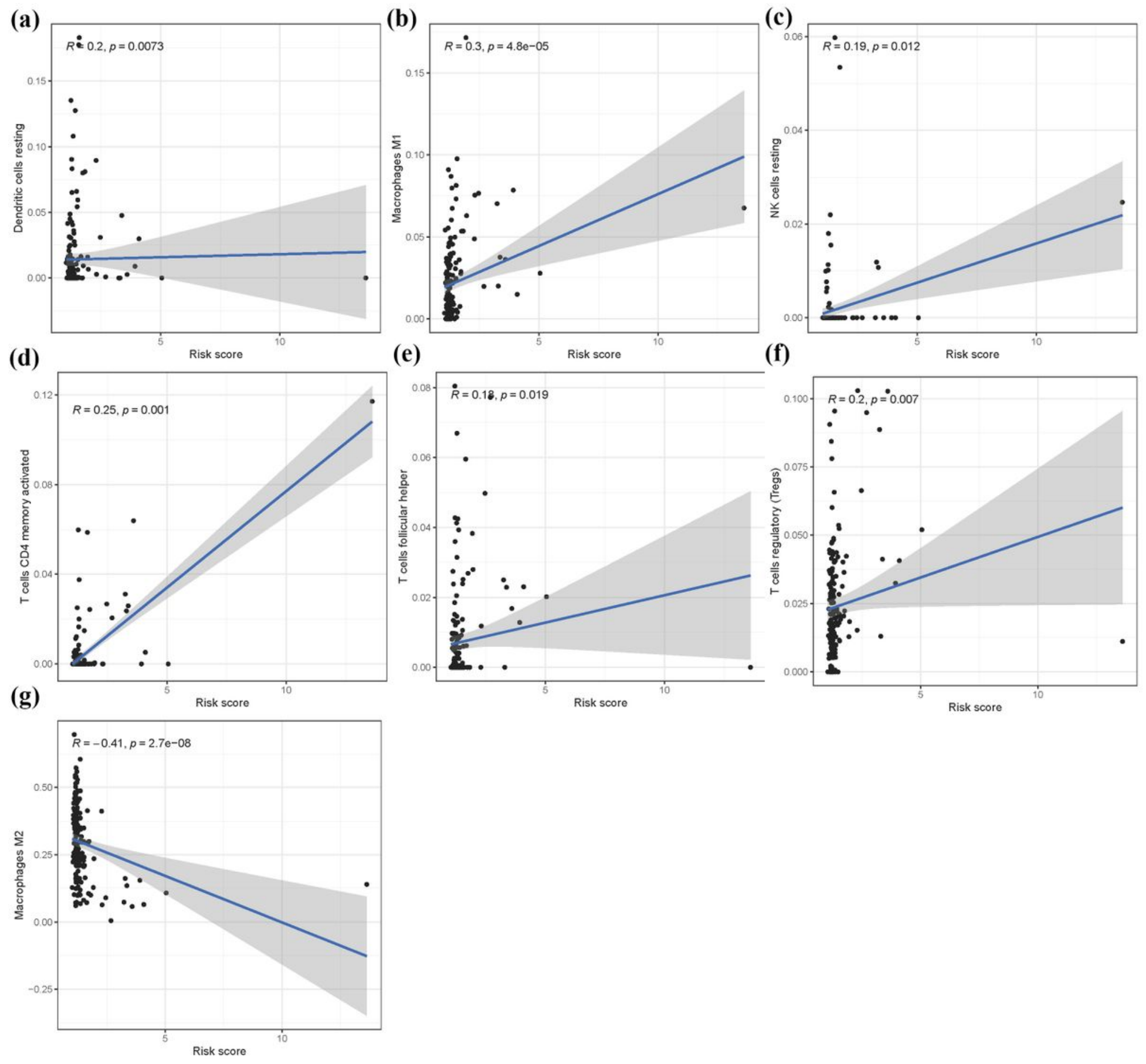

Figure 11

Scatterplot of correlation analysis of risk score and immune cells. (a): Dendritic cells resting. (b): Macrophages M1. (c): NK cells resting. (d): T cells CD4 memory activated. (e): T cells follicular helper. (f): T cells regulatory (Tregs). (g): Macrophages M2. 
Risk 追 low 追 high

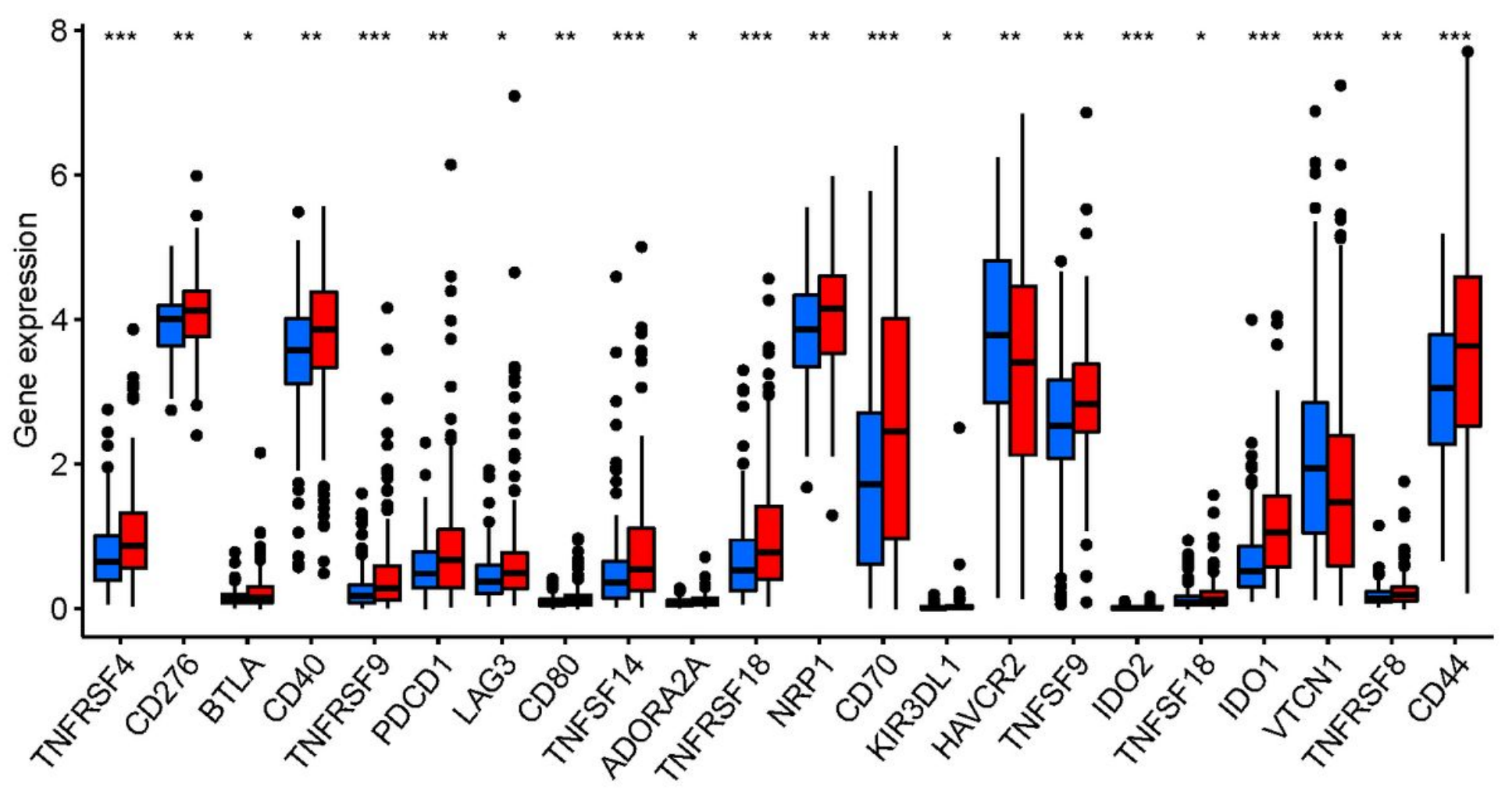

Figure 12

Immune checkpoint expression. 